\title{
Stellar SEDs from 0.3 to 2.5 ?m: Tracing the Stellar Locus and Searching for Color Outliers in the SDSS and 2MASS
}

\section{Citation}

Covey, K. R., Z. Ivezic, D. Schlegel, D. Finkbeiner, N. Padmanabhan, R. H. Lupton, M. A. Agüeros, et al. 2007. "Stellar SEDs from 0.3 to 2.5 ?m: Tracing the Stellar Locus and Searching for Color Outliers in the SDSS and 2MASS." The Astronomical Journal 134 (6) (November 6): 2398-2417. doi:10.1086/522052.

\section{Published Version}

doi:10.1086/522052

\section{Permanent link}

http://nrs.harvard.edu/urn-3:HUL.InstRepos:33461898

\section{Terms of Use}

This article was downloaded from Harvard University's DASH repository, and is made available under the terms and conditions applicable to Other Posted Material, as set forth at http:// nrs.harvard.edu/urn-3:HUL.InstRepos:dash.current.terms-of-use\#LAA

\section{Share Your Story}

The Harvard community has made this article openly available.

Please share how this access benefits you. Submit a story.

Accessibility 


\title{
STELLAR SEDs FROM 0.3 TO $2.5 \mu \mathrm{m}$ : TRACING THE STELLAR LOCUS AND SEARCHING FOR COLOR OUTLIERS IN THE SDSS AND 2MASS
}

\author{
K. R. Covey, ${ }^{1}$ Ž. Ivezić, ${ }^{2}$ D. Schlegel, ${ }^{3}$ D. Finkbeiner, ${ }^{1}$ N. Padmanabhan, ${ }^{3}$ R. H. Lupton, ${ }^{4}$ M. A. Agüeros, ${ }^{5}$ \\ J. J. Bochanski, ${ }^{2}$ S. L. Hawley, ${ }^{2}$ A. A. West, ${ }^{6}$ A. Seth, ${ }^{1}$ A. Kimball, ${ }^{2}$ S. M. Gogarten, ${ }^{2}$ \\ M. Clatre, ${ }^{2}$ D. Haggard, ${ }^{2}$ N. Kaib, ${ }^{2}$ D. P. Schneider, ${ }^{6}$ and B. Sesar ${ }^{2}$ \\ Received 2007 May 6; accepted 2007 July 26
}

\begin{abstract}
The Sloan Digital Sky Survey (SDSS) and Two Micron All Sky Survey (2MASS) are rich resources for studying stellar astrophysics and the structure and formation history of the Galaxy. As new surveys and instruments adopt similar filter sets, it is increasingly important to understand the properties of the ugrizJHK $K_{S}$ stellar locus, both to inform studies of "normal" main-sequence stars and enable robust searches for point sources with unusual colors. Using a sample of $\sim 600,000$ point sources detected by SDSS and 2MASS, we tabulate the position and width of the ugrizJHK stellar locus as a function of $g-i$ color, and provide accurate polynomial fits. We map the Morgan-Keenan spectral type sequence to the median stellar locus by using synthetic photometry of spectral standards and by analyzing 3000 SDSS stellar spectra with a custom spectral typing pipeline, described in the Appendix to this paper. We develop an algorithm to calculate a point source's minimum separation from the stellar locus in a seven-dimensional color space, and use it to robustly identify objects with unusual colors, as well as spurious SDSS/2MASS matches. Analysis of a final catalog of 2117 color outliers identifies 370 white-dwarf/M dwarf(WDMD) pairs, 93 QSOs, and $90 \mathrm{M}$ giant/carbon star candidates, and demonstrates that WDMD pairs and QSOs can be distinguished on the basis of their $J-K_{s}$ and $r-z$ colors. We also identify a group of objects with correlated offsets in the $u-g$ versus $g-r$ and $g-r$ versus $r-i$ color-color spaces, but subsequent follow-up is required to reveal the nature of these objects. Future applications of this algorithm to a matched SDSS-UKIDSS catalog may well identify additional classes of objects with unusual colors by probing new areas of color-magnitude space.
\end{abstract}

Key words: Galaxy: stellar content — infrared: stars — stars: early-type — stars: late-type — surveys

Online material: color figures, machine-readable tables, tar file

\section{INTRODUCTION}

The Two Micron All Sky Survey (2MASS; Skrutskie et al. 1997) and Sloan Digital Sky Survey (SDSS; York et al. 2000) are fertile grounds for identifying rare stellar objects with unusual colors, such as brown dwarfs (Kirkpatrick et al. 1999; Strauss et al. 1999; Burgasser et al. 1999), carbon stars (Margon et al. 2002), RR Lyrae stars (Ivezić et al. 2005), and white dwarf/M dwarf( WDMD) pairs (Raymond et al. 2003; Smolčić et al. 2004; Silvestri et al. 2006). Relatively little attention has been devoted to understanding and documenting the detailed characteristics of "normal" mainsequence stars detected in these surveys, despite the fact that they represent an important source of contamination in the search for any set of rare objects.

Recent studies have also demonstrated the power of combining survey data at differing wavelengths to provide insights into the nature of various astrophysical sources. Finlator et al. (2000) showed that population synthesis models of the Galaxy, based on Kurucz (1979) models, can reproduce the colors of the eightband SDSS/2MASS stellar locus (except for stars colder than

\footnotetext{
${ }^{1}$ Harvard Smithsonian Center for Astrophysics, 60 Garden Street, Cambridge, MA 02138,USA; kcovey@cfa.harvard.edu.

2 Department of Astronomy, University of Washington, Box 351580, Seattle, WA 98195, USA.

3 Lawrence Berkeley National Laboratory, 1 Cyclotron Road, Mail Stop 50R5032, Berkeley, CA 94720, USA.

4 Princeton University Observatory, Princeton, NJ 08544, USA.

5 NSF Fellow; Department of Astronomy, Columbia University, 550 West 120th Street, New York, NY 10027, USA.

${ }^{6}$ Astronomy Department, University of California Berkeley, 601 Campbell Hall, Berkeley, CA 94720-3411, USA
}

$\sim 3000 \mathrm{~K})$. Agüeros et al. (2005) analyzed a catalog of $G A L E X$ SDSS sources, constraining the fraction of GALEX sources with optical counterparts and investigating the ability of GALEX and $G A L E X$-SDSS catalogs to distinguish between various classes of UV-bright objects (white dwarfs, starburst galaxies, active galactic nuclei, etc.), while Anderson et al. (2007) have combined the ROSAT and SDSS catalogs to construct the largest sample of $\mathrm{X}$-ray-luminous QSOs to date, including hundreds of rare objects, such as X-ray-emitting BL Lacertae objects. West (2007) used a matched SDSS- $-\mathrm{H}$ I data set to investigate the relationship between the stellar population, neutral gas content, and star formation history of a homogeneous set of galaxies spanning a wide range of masses and morphologies. By supplementing the "main" SDSS sample of galaxies with observations from surveys ranging from the X-ray (ROSAT) to the radio (FIRST, NVSS), Obric et al. (2006) demonstrated that the optical/near-infrared (NIR) spectral energy distributions (SEDs) of galaxies are remarkably uniform; the $K$-band flux of a galaxy can be predicted with an accuracy of 0.1 mag based solely on its $u-r$ color, redshift, and estimated dust content. These studies show that the union of data sets derived from surveys covering differing wavelength ranges allow investigations that would be impossible using either data set in isolation.

While the main science drivers of SDSS-I were extragalactic in nature, the SEGUE component of SDSS-II is extending the survey footprint through the Galactic plane. In addition, several other surveys are now or will shortly be mapping much of the sky in the optical (the Panoramic Survey Telescope and Rapid Response System [Pan-STARRS; Kaiser et al. 2002] and SkyMapper [Keller et al. 2007]) and NIR (the United Kingdom Infrared Deep Sky Survey [UKIDSS; Warren et al. 2007]) using filter systems that 
closely resemble those of SDSS and 2MASS. As a result, these surveys are increasingly useful to anyone who wishes to identify and study stellar objects.

To aid in the study of point sources identified by these surveys, we present a detailed characterization of the optical/NIR properties of stars detected by both SDSS and 2MASS, as well as an algorithm for identifying point sources with optical/NIR colors that differ significantly from those of typical main-sequence stars. In $\S 2$ we describe the assembly of our matched SDSS/ 2MASS stellar catalog. We provide a detailed characterization of the locus of main-sequence stars in SDSS/2MASS color-color space in $\S 3$. We give a robust algorithm for identifying color outliers from the stellar locus in $\S 4$, along with a description of the resultant catalog generated by the application of this algorithm to our data set. We summarize our work and highlight our conclusions in $\S 5$.

\section{ASSEMBLING A CATALOG OF MATCHED SDSS/2MASS POINT SOURCES}

\subsection{The Sloan Digital Sky Survey}

The SDSS has imaged nearly a quarter of the sky, centered on the north Galactic cap, at optical wavelengths (York et al. 2000). The latest public Data Release (DR5) includes photometry for $2.1 \times 10^{8}$ unique objects over $\sim 8000 \mathrm{deg}^{2}$ of sky (AdelmanMcCarthy et al. 2007). Since the completion of the survey's initial mission, new scientific studies (collectively known as SDSS-II) have begun to add new spatial and temporal coverage to the SDSS database. ${ }^{7}$ The SDSS camera (Gunn et al. 1998), populated by six CCDs in each of five filters $(u, g, r, i, z$; Fukugita et al. 1996) observes in time delay and integrate mode to generate near-simultaneous photometry along a strip $2.5^{\circ}$ in width; a second scan, slightly offset from the first, fills in the areas on the sky that fall in the gaps between CCDs. The survey's photometric calibration strategy (Hogg et al. 2001; Smith et al. 2002; Tucker et al. 2006) produces a final catalog which is $95 \%$ complete to a depth of $r \sim 22.2$, and accurate to $0.02 \mathrm{mag}$ (both absolute and rms error) for sources not limited by Poisson statistics (Ivezić et al. 2004). Sources with $r<20.5$ have astrometric errors less than $0.1^{\prime \prime}$ per coordinate (rms; Pier et al. 2003), and robust star/galaxy separation is achieved above $r \sim 21.5$ (Lupton et al. 2001).

SDSS photometry provides candidates for spectroscopic observation with the SDSS twin fiber-fed spectrographs (Newman et al. 2004), mounted on the same dedicated $2.5 \mathrm{~m}$ telescope (Gunn et al. 2006) as the survey's imaging camera. The spectrographs give wavelength coverage from 3800 to $9200 \AA$ with a resolution of $\lambda / \Delta \lambda \sim 1800$. Each fiber has a $3^{\prime \prime}$ diameter and is plugged into a hole in a predrilled metal plate allowing observations across a $3^{\circ}$ field. A single plate accommodates 640 fibers (320 going to each spectrograph), of which $\approx 50$ are typically reserved for calibration purposes. The SDSS spectroscopic catalog contains over $10^{6}$ objects, including $6 \times 10^{5}$ galaxies, $1.5 \times 10^{5}$ stars, and nearly $8 \times 10^{4}$ quasars (Schneider et al. 2007).

\subsection{The Two Micron All Sky Survey}

The Two Micron All Sky Survey used two $1.3 \mathrm{~m}$ telescopes to survey the entire sky in near-infrared light (Skrutskie et al. 1997; Cutri et al. 2003). ${ }^{8}$ Each telescope's camera was equipped with three $256 \times 256$ arrays of $\mathrm{HgCdTe}$ detectors with $2^{\prime \prime}$ pixels and observed simultaneously in the $J(1.25 \mu \mathrm{m}), H(1.65 \mu \mathrm{m})$, and

\footnotetext{
${ }^{7}$ See http://www.sdss.org.

${ }^{8}$ See http://www.ipac.caltech.edu/2mass.
}

$K_{s}(2.17 \mu \mathrm{m})$ bands. The detectors were sensitive to point sources brighter than about $1 \mathrm{mJy}$ at the $10 \sigma$ level, corresponding to limiting (Vega-based) magnitudes of $15.8,15.1$, and 14.3, respectively. Point-source photometry is repeatable to better than $10 \%$ precision at this level, and the astrometric uncertainty for these sources is less than $0.2^{\prime \prime}$. The 2MASS catalogs contain positional and photometric information for $\sim 5 \times 10^{8}$ point sources and $\sim 2 \times 10^{6}$ extended sources.

\subsection{The Merged Sample}

We have assembled a sample of point sources from the SDSS DR2 catalog (Abazajian et al. 2004) using data processed by the photometric pipeline described by Padmanabhan et al. (2007) and Finkbeiner et al. (2004b). In this reduction pipeline SDSS detections are automatically matched to the nearest 2MASS source within $3^{\prime \prime}$ of the astrometric position of the SDSS detection. To ensure our analysis is not unduly affected by sources with poor photometry, we selected for analysis in this work only those objects which meet the following criteria:

1. Unblended, unsaturated, and accurate 2MASS photometry: $K_{s}<14.3$ (the 2MASS completeness limit), read flag (rd_flg) = 2 , blend flag (bl_flg) $=1$, and contamination and confusion flag (cc_flg) $=0$ in $J, H$, and $K_{s}{ }^{9}{ }^{9}$

2. Unblended, unsaturated, reliable SDSS photometry of stars: $i<21.3$ (the SDSS 95\% completeness limit), BLENDED flag $=0$, DEBLENDED_AS_MOVING flag $=0$, SATURATED flag $=0$, PRIMARY flag $=1$, INTERP_CENTER $=0$, EDGE $=$ 0 , SATURATED_CENTER $=0$, PSF_FLUX_INTERP $=0$, and object type $=$ STAR ${ }^{10}$

The catalog resulting from these cuts contains 687,150 point sources above the $K_{s}<14.3$ effective flux limit. Each object in the sample has measurements in eight filters (SDSS ugriz, 2MASS $J H K_{s}$ ) stretching from $3000 \AA$ to $2.5 \mu \mathrm{m}$; the footprint of the sample is shown in Figure 1. As we seek to study point sources, we adopted point-spread function (PSF) magnitudes for SDSS sources, without correcting for Galactic extinction; as the Schlegel et al. (1998) dust map measures the total Galactic extinction along the line of sight, it may overestimate the true extinction to nearby stars. In any event, we expect the impact of applying extinction corrections to our sample would be small; as the effect of extinction on infrared colors is small, and the reddening vector is nearly parallel to the stellar locus in SDSS color space (Finkbeiner et al. 2004a), objects with uncorrected extinction will merely be shifted along, and not out of, the standard stellar locus.

\section{DEFINING THE STANDARD STELLAR LOCUS}

\subsection{The High-Quality Sample}

To ensure the sample used to determine the basic shape of the typical stellar locus is of high photometric quality, we identify a subset of our catalog that satisfies the following additional quality cuts:

1. Colors minimally affected by reddening from the interstellar medium: $A_{r}<0.2$ as estimated by the dust map of Schlegel et al. (1998).

2. Acceptable photometric conditions: SDSS field quality flag (fieldQA) $>0$.

3. Isolated sources: SDSS CHILD flag $=0$. (When a single photometric detection appears to be made up of multiple overlapping

\footnotetext{
${ }^{9}$ See http://www.ipac.caltech.edu/2mass/releases/allsky/doc/explsup.html for a full description of 2MASS flags.

${ }^{10}$ See Stoughton et al. (2002) for a full description of SDSS flags.
} 


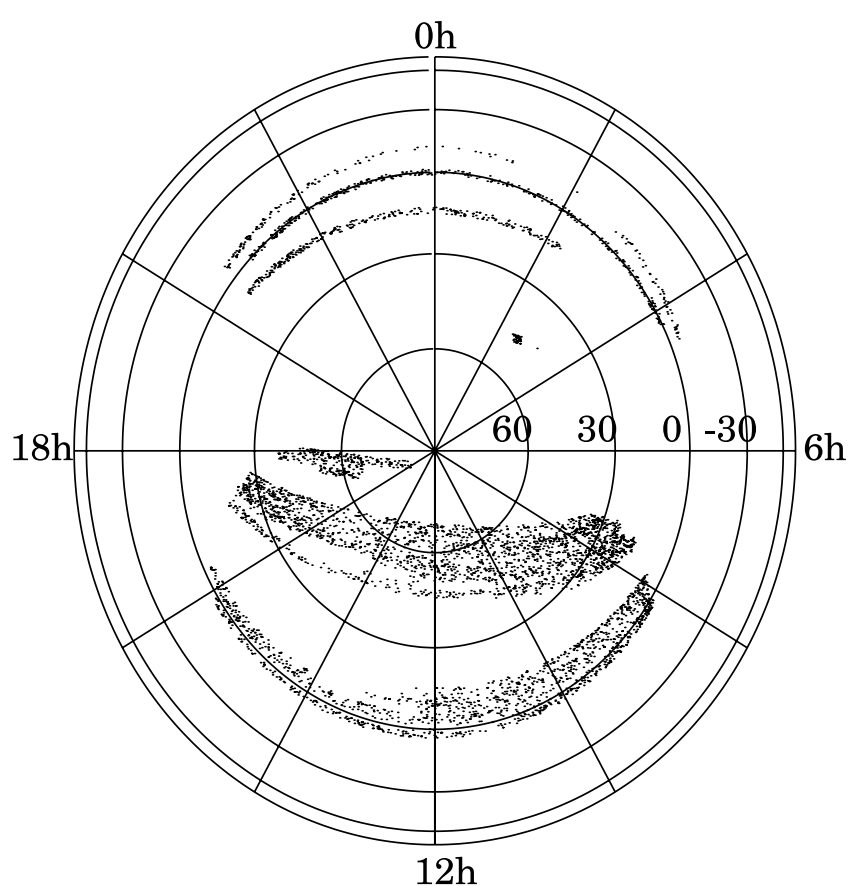

FIG. 1.-Footprint covered by the matched SDSS/2MASS catalog, represented in an equal-area polar projection (in right ascension and declination coordinates). For computational efficiency, the footprint is shown using an $\sim 0.5 \%$ subset of the full matched SDSS-2MASS data set.

objects, the SDSS photometric pipeline uses a process known as "deblending" 11 to separate the individual components selfconsistently in all filters, and sets the CHILD flag for all components.)

4. Minimal chance of spurious catalog matches: SDSS and 2MASS positional separation $<0.6^{\prime \prime}$.

These cuts produce a subsample of 311,652 objects that we refer to as the "high-quality" sample.

Figure 2 displays the locations of stars in the high-quality sample within color-color and color-magnitude space. We use "native" magnitudes to construct these diagrams; note that SDSS uses an AB-based magnitude system, while 2MASS uses a Vegabased magnitude system (Finlator et al. [2000], however, provide the $J H K_{s}$ offsets required to place 2MASS magnitudes on an AB system). The first six panels display color-color diagrams constructed using the seven "adjacent colors" (a phrase which we use as shorthand for the complete set of $u-g, g-r, r-i, i-z$, $z-J, J-H$, and $H-K_{s}$ ). The stellar locus is clearly visible in these diagrams, typically extending from the bluest stars (in the lower left corner in all panels) to the reddest stars (in the upper right of all panels). ${ }^{12}$

The seventh panel of Figure 2 displays a $g-i$ versus $i-K_{S}$ color-color diagram, which samples as broad a range in wavelength as is possible without including $u$-band measurements, which are less reliable for red stars due to their low intrinsic flux and a "red leak" in the SDSS camera. The red leak stems from flux at $7100 \AA$ passing through the $u$-band filter due to a change in the filter's interference coating under vacuum. This causes a color-dependent offset to $u$-band photometry of $\sim 0.02$ mag for $\mathrm{K}$ stars, $\sim 0.06 \mathrm{mag}$ for M0 stars, and $0.3 \mathrm{mag}$ for stars with

\footnotetext{
11 See http://www.sdss.org/dr5/algorithms/deblend.html for a full discussion of the SDSS deblending algorithm.

${ }^{12}$ We have chosen to display these color-color diagrams with the bluer color on the $y$-axis and the redder color on the $x$-axis, as is customary for stellar astronomers; this differs from the standard SDSS convention, in which color-color diagrams typically display the bluer color on the $x$-axis and the redder color on the $y$-axis.
}

$r-i \sim 1.5$. The effect depends on a star's instrumental $u$ and $r$ magnitudes, which are sensitive to air mass, seeing, and the detailed interaction between the $u$ filter in each camera column and the sharp molecular features in the spectra of red stars. The standard SDSS reduction does not attempt to correct for this effect, resulting in a large $u-g$ dispersion at the red end of the stellar locus (as seen in the $u-g$ vs. $g-r$ color-color diagram in Fig. 2), even for a high-quality sample such as this one.

In the eighth panel of Figure 2 we show the distribution of our sample in $g-i$ versus $i$ color-magnitude space. The faint magnitude limit of the sample is clearly apparent, ranging from $i \sim 16.3$ at $g-i=1$ to $i \sim 17.7$ at $g-i=3$. This faint limit is a result of selecting only those SDSS sources with well-measured 2MASS counterparts, thus requiring that $K_{s}<14.3$. Redder stars are fainter in $i$ for a given $K_{s}$ magnitude, and thus a constant $K_{S}$ faint limit results in an effective color-dependent $i$-band faint limit, such that stars with $g-i \sim 1$ must have $i<16.3$ to be detected in 2MASS, while stars with $g-i \sim 3$ can be more than a magnitude fainter $(i<17.7)$.

The SDSS catalog contains $\mathrm{S} / \mathrm{N}=10$ detections down to $i=$ 21.5 , and Figure 2 demonstrates that our sample is nearly entirely composed of sources brighter than $i \sim 19$; requiring a 2MASS counterpart has biased our sample toward the brightest of the stars detected by SDSS. Our primary goals, however, are to explore the areas of color-space populated by astronomical point sources, not to compare the relative numbers of objects in those areas; this bias does not seriously compromise the effectiveness of our study. It does affect our sensitivity to the least luminous, reddest members of the stellar locus (very low mass stars and brown dwarfs), which we can observe only in a very small volume centered on the Sun. To the extent that these objects are extremely hard to detect within the completeness limits of the two surveys, our high-quality sample does not allow characterization of the behavior of the stellar locus beyond the location of mid-M type objects. The $K$-band flux limit also preferentially selects nearby disk stars, resulting in a sample dominated by relatively metalrich stars.

\subsection{The Median Stellar Locus}

In order to identify sources in unusual areas of color-color space, we must first characterize the properties of the stellar locus, where the vast majority of stars are found. To first order, the observational properties of main-sequence stars can be characterized by their photospheric temperature, or $T_{\text {eff }}$. In addition, most stellar colors become monotonically redder as $T_{\text {eff }}$ decreases, ${ }^{13}$ allowing a single color to serve as a useful parameterization for the majority of the variance in stellar colors.

We choose to parameterize the stellar locus as a function of $g-i$ color because it will be applicable to the entire SDSS catalog, not merely the subset with 2MASS counterparts, and because it samples the largest wavelength range possible without relying on shallower $u$ or $z$ measurements. As can be seen in Figure 2 (bottom right), our sample spans more than 4 mag of $g-i$ color with typical $g-i$ errors $\sim 0.03 \mathrm{mag}$. The range and precision of the $g-i$ color allow the separation of stars into more than 130 independent $g-i$ bins, in principle a more precise classification than allowed by Morgan-Keenan (MK) spectral types. The $g-i$ color most efficiently identifies objects whose effective temperatures $\left(T_{\text {eff }}\right)$ place the peak of their blackbody emission within the wavelength range spanned by the $g$ and $i$ filters, $4000-8200 \AA$. As a result, the $g-i$ color will be most sensitive to selecting objects with $3540 \mathrm{~K}<$ $T_{\text {eff }}<7200 \mathrm{~K}$, corresponding roughly to spectral types from $\mathrm{F}$ to

\footnotetext{
13 This is not uniformly true, however; $J-H$ becomes bluer with decreasing $T_{\text {eff }}$ for $\mathrm{M}$ stars, for example.
} 

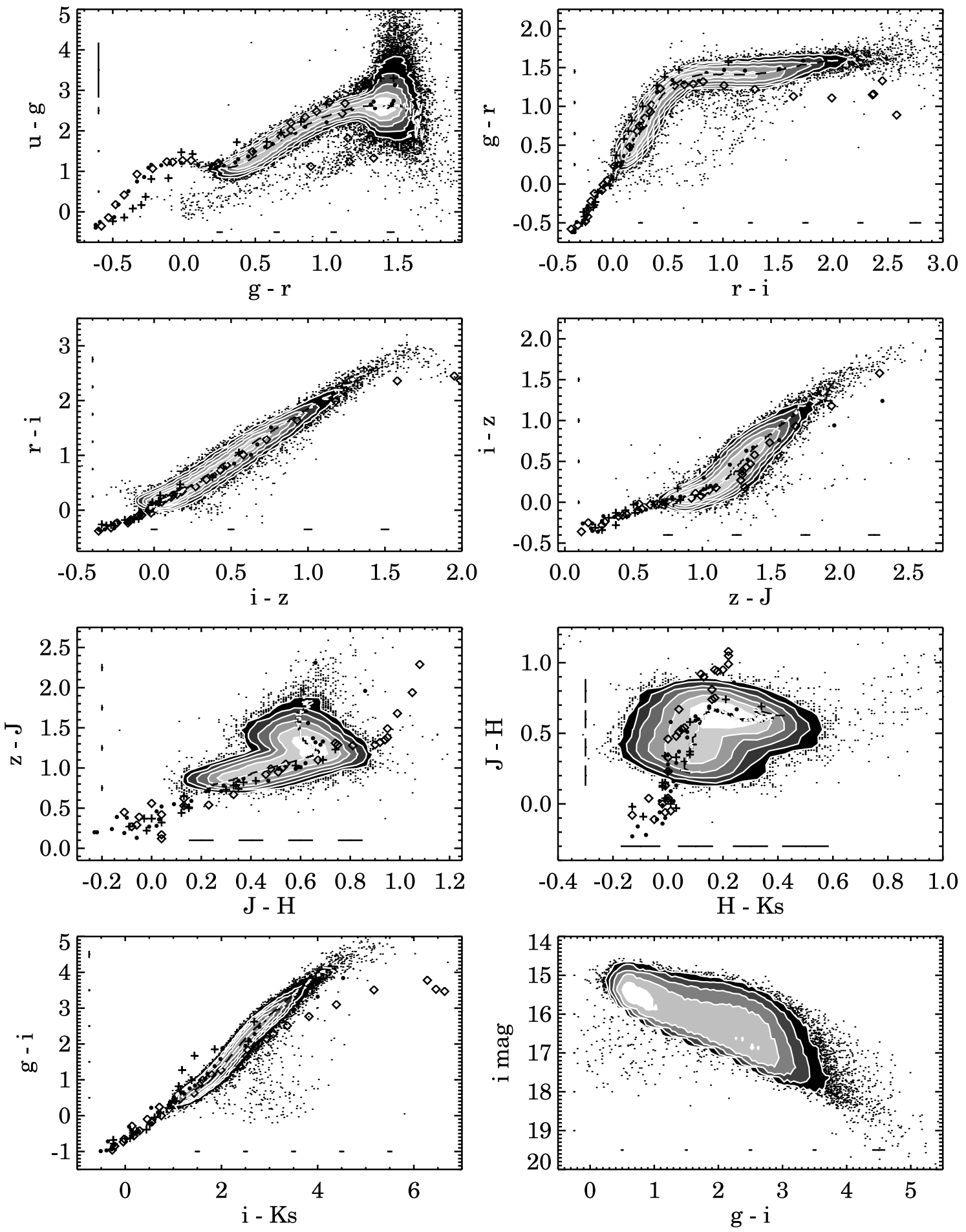

FIG. 2.-Color-color and color-magnitude diagrams showing the density of objects in the high-quality SDSS/2MASS sample described in $\S 3.1$. Black dots show individual sources; contours show source density in saturated regions. Contours begin at source densities of 10,000 and 2000 sources per square magnitude in color-color and color-magnitude space, respectively; steps between contours indicate an increase in source density by a factor of 3 . The black and white dashed line shows the location of the median stellar locus, calculated in $\S 3.2$ and tabulated in Table 1. Error bars near the axes of each diagram show the median photometric error as a function of color and magnitude. Symbols show the synthetic SDSS/2MASS colors of solar-metallicity Pickles (1998) spectral standards, as calculated in $\S 3.3$ and given in Table 3; dwarf stars are shown as dots, and giants and supergiants are shown as diamonds and crosses, respectively. [See the electronic edition of the Journal for a color version of this figure.]

mid-M. Other colors (such as $i-z$ ) allow for precise separation of the stellar locus as a function of $T_{\mathrm{eff}}$ outside this temperature range.

The first three panels of Figure 3 show the $u-g, r-i$, and $H-K_{s}$ colors of the stellar locus as a function of $g-i$. To parameterize the stellar locus, we have measured the median value of each adjacent color in $g-i$ bins in the range $0.10<g-$ $i<4.34$. The standard bin width (and spacing) is $0.02 \mathrm{mag}$, with two expanded bins of $0.1 \mathrm{mag}$ at each end of the $g-i$ range (necessary to extend the median locus into areas of low stellar density). We present the median value and dispersion of each adjacent color as a function of $g-i$ in Table 1 . We use the interquartile width to characterize the width of the locus, as it is a more robust measure of a distribution's width than the standard deviation in the presence of large, non-Gaussian outliers. Following convention, 

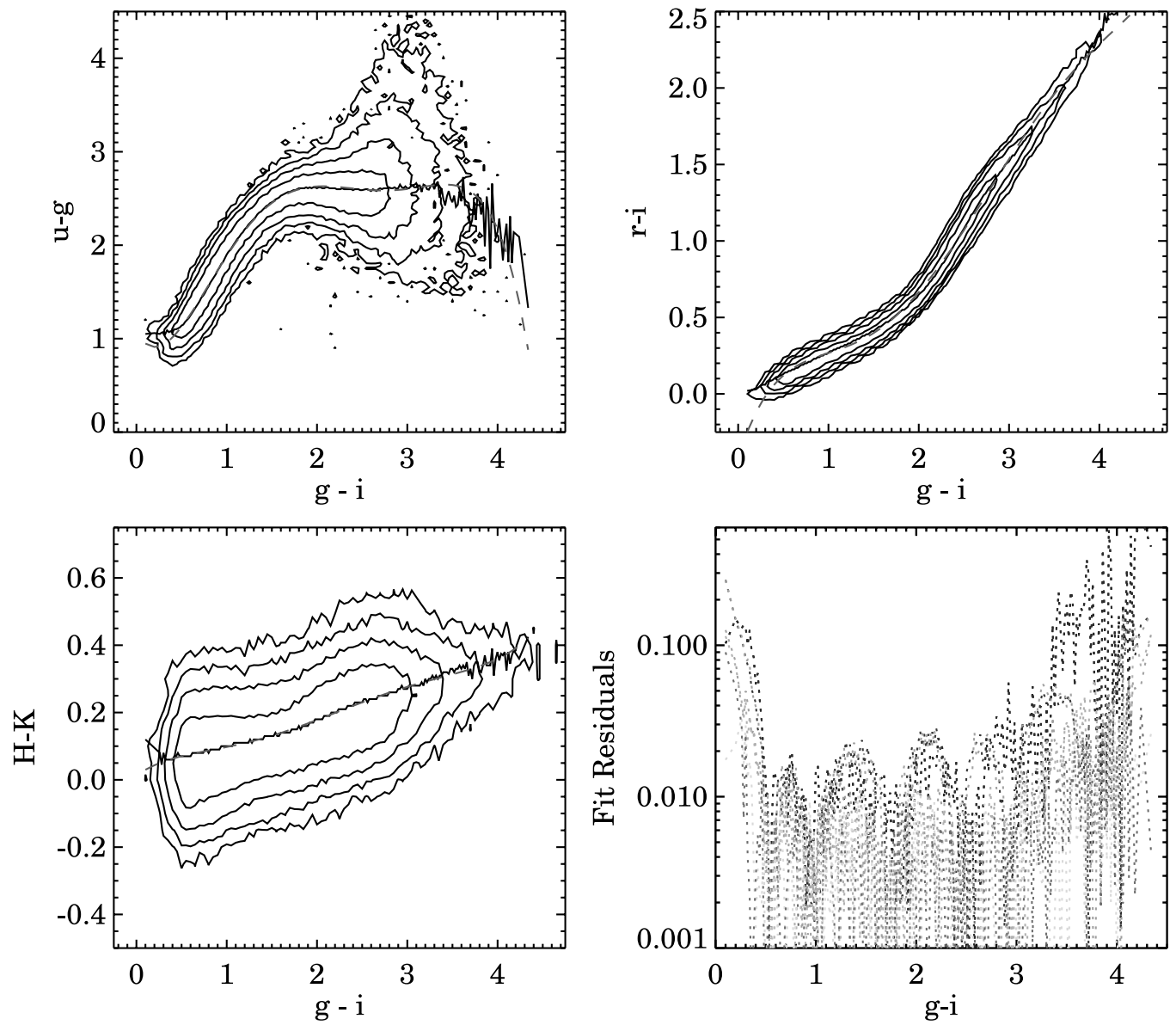

FIG. 3.-The $u-g$ (top left), $r-i$ (top right), and $H-K_{s}$ (bottom left) colors as a function of $g-i$. Stellar density in each color-color diagram is shown with contours; contour steps indicate a factor of 5 increase in density. The median value of each color as a function of $g-i$ is shown with a solid line; dashed lines show analytic fits to the median values using the coefficients given in Table 2. Bottom right: Residuals between the median value of each adjacent color and the corresponding analytic fit. The color of each line represents the mean wavelength of the adjacent color whose residuals are shown, such that $u-g$ residuals are shown in black and $H-K_{s}$ residuals are shown in dark gray. [See the electronic edition of the Journal for a color version of this figure.]

however, we present in Table 1 "pseudo-standard deviations," calculated as $74 \%$ of the interquartile width, using the relation between the interquartile width and standard deviation for a wellbehaved Gaussian distribution. We do not explicitly include the uncertainty in the median value of each adjacent color, but it can easily be calculated by dividing the pseudo-standard deviation of each bin by the square root of the number of objects in the bin (also given in Table 1). The median values of $u-g, r-i$, and $H-K_{s}$ are shown in Figure 3 as a function of $g-i$, with the location of the parameterized locus in color-color space shown in Figure 2.
We have fit the adjacent colors of the median stellar locus using a fifth-order polynomial in $g-i$, with the form

$$
\text { color } X=\sum_{k=0}^{5} A_{k}(g-i)^{k} \text {. }
$$

The resulting fits are compared to the median $u-g, r-i$, and $H-K_{s}$ colors in the first three panels of Figure 3, and the coefficients used to produce each fit are presented in Table 2. The last two columns of Table 2 provide a measure of the accuracy

TABLE 1

Locus Colors as a Function of $g-i$ Color

\begin{tabular}{|c|c|c|c|c|c|c|c|c|c|c|c|c|c|c|c|}
\hline$g-i$ & $n_{\text {stars }}$ & $u-g$ & $\sigma_{u-g}$ & $g-r$ & $\sigma_{g-r}$ & $r-i$ & $\sigma_{r-i}$ & $i-z$ & $\sigma_{i-z}$ & $z-J$ & $\sigma_{z-J}$ & $J-H$ & $\sigma_{J-H}$ & $H-K_{s}$ & $\sigma_{H-K_{s}}$ \\
\hline $0.10 \ldots \ldots \ldots \ldots \ldots \ldots$ & 25 & 1.05 & 0.67 & 0.09 & 0.04 & 0.02 & 0.02 & -0.03 & 0.06 & 0.76 & 0.19 & 0.23 & 0.38 & 0.12 & 0.51 \\
\hline $0.20 \ldots \ldots \ldots \ldots \ldots \ldots$ & 70 & 1.06 & 0.10 & 0.18 & 0.03 & 0.03 & 0.03 & -0.02 & 0.06 & 0.77 & 0.07 & 0.22 & 0.07 & 0.09 & 0.11 \\
\hline $0.26 \ldots \ldots \ldots \ldots \ldots \ldots$ & 15 & 1.05 & 0.05 & 0.21 & 0.01 & 0.06 & 0.01 & -0.03 & 0.02 & 0.77 & 0.03 & 0.22 & 0.04 & 0.08 & 0.01 \\
\hline $0.28 \ldots \ldots \ldots \ldots \ldots \ldots$ & 50 & 1.07 & 0.07 & 0.22 & 0.02 & 0.06 & 0.02 & -0.03 & 0.03 & 0.77 & 0.04 & 0.24 & 0.05 & 0.05 & 0.08 \\
\hline $0.30 \ldots \ldots \ldots \ldots \ldots \ldots$ & 49 & 1.07 & 0.07 & 0.24 & 0.03 & 0.06 & 0.03 & 0.00 & 0.04 & 0.79 & 0.04 & 0.23 & 0.06 & 0.08 & 0.10 \\
\hline $0.32 \ldots \ldots \ldots \ldots \ldots \ldots$ & 132 & 1.05 & 0.10 & 0.25 & 0.03 & 0.08 & 0.02 & 0.00 & 0.04 & 0.78 & 0.04 & 0.25 & 0.06 & 0.06 & 0.09 \\
\hline $0.34 \ldots \ldots \ldots \ldots \ldots$ & 86 & 1.09 & 0.09 & 0.26 & 0.02 & 0.09 & 0.02 & 0.00 & 0.04 & 0.80 & 0.04 & 0.25 & 0.07 & 0.06 & 0.07 \\
\hline $0.36 \ldots \ldots \ldots \ldots \ldots$ & 212 & 1.07 & 0.10 & 0.28 & 0.03 & 0.09 & 0.02 & 0.00 & 0.03 & 0.80 & 0.04 & 0.26 & 0.05 & 0.06 & 0.07 \\
\hline $0.38 \ldots \ldots \ldots \ldots \ldots$ & 178 & 1.08 & 0.07 & 0.29 & 0.03 & 0.09 & 0.03 & 0.01 & 0.04 & 0.81 & 0.04 & 0.28 & 0.05 & 0.06 & 0.08 \\
\hline $0.40 \ldots \ldots \ldots \ldots \ldots$ & 442 & 1.07 & 0.09 & 0.30 & 0.02 & 0.10 & 0.03 & 0.01 & 0.03 & 0.81 & 0.04 & 0.28 & 0.06 & 0.06 & 0.07 \\
\hline
\end{tabular}

NotEs.-Table 1 is published in its entirety in the electronic edition of the Astronomical Journal. A portion is shown here for guidance regarding its form and content. 
TABLE 2

Analytic Fits to the Stellar Locus

\begin{tabular}{|c|c|c|c|c|c|c|c|c|}
\hline Color & $A_{0}$ & $A_{1}$ & $A_{2}$ & $A_{3}$ & $A_{4}$ & $A_{5}$ & Max. Res. & Med. Res. \\
\hline$u-g \ldots \ldots \ldots \ldots$ & 1.0636113 & -1.6267818 & 4.9389572 & -3.2809081 & 0.8725109 & -0.0828035 & 0.6994 & 0.0149 \\
\hline$g-r \ldots \ldots \ldots \ldots$ & 0.4290263 & -0.8852323 & 2.0740616 & -1.1091553 & 0.2397461 & -0.0183195 & 0.2702 & 0.0178 \\
\hline$r-i \ldots \ldots \ldots \ldots \ldots$ & -0.4113500 & 1.8229991 & -1.9989772 & 1.0662075 & -0.2284455 & 0.0172212 & 0.2680 & 0.0164 \\
\hline$i-z \ldots \ldots \ldots \ldots \ldots$ & -0.2270331 & 0.7794558 & -0.7350749 & 0.3727802 & -0.0735412 & 0.0049808 & 0.1261 & 0.0071 \\
\hline$z-J \ldots \ldots \ldots \ldots \ldots$ & 0.5908002 & 0.5550226 & -0.0980948 & -0.0744787 & 0.0416410 & -0.0051909 & 0.1294 & 0.0054 \\
\hline$J-H \ldots \ldots \ldots \ldots$ & 0.2636025 & -0.2509140 & 0.9660369 & -0.6294004 & 0.1561321 & -0.0134522 & 0.0413 & 0.0042 \\
\hline$H-K \ldots \ldots \ldots \ldots$ & 0.0134374 & 0.1844403 & -0.1989967 & 0.1300435 & -0.0344786 & 0.0032191 & 0.0900 & 0.0040 \\
\hline
\end{tabular}

of each fit by presenting the maximum and median offsets between the median stellar locus and each analytic description; residuals between the median adjacent colors and the corresponding analytic fit are shown as a function of $g-i$ in the fourth panel of Figure 3 . These polynomial relations match the actual median behavior well (typical residuals $\leq 0.02 \mathrm{mag}$ ). As we weighted points along the median locus proportionally to the number of stars in each $g-i$ bin, the fits perform better in densely populated color regions $(0.5<g-i<3.0)$, and more poorly at the sparsely populated blue and red ends.

We note that while we have restricted our catalog to objects identified as point sources by the SDSS reduction pipeline, contamination by extragalactic sources or unresolved multiple stars cannot be prevented completely, and could bias this measurement of the median stellar locus. Ivezić et al. (2002) identify distinct color differences in the optical/NIR colors of stars, galaxies, and QSOs (see their Fig. 3); only 229 of the 311,652 high-quality sources (or $0.07 \%$ ) analyzed here lie in the region of color-color space $\left(i-K_{s}>1.5+0.75 \times g-i\right)$ occupied by extragalactic sources, regardless of their morphological classification. This is partially explained by the de facto magnitude limit imposed on our sample by requiring 2 MASS detections; $98.7 \%$ of the highquality sample is brighter than $r=19$, the magnitude at which galaxies begin to outnumber stars.

Unresolved binaries surely represent a larger source of contamination for a sample such as this, with estimates of the stellar multiplicity fraction ranging from $57 \%$ for $\mathrm{G}$ type stars (Duquennoy \& Mayor 1991) to 30\% for M type stars (Delfosse et al. 2004). Indeed, Pourbaix et al. (2005) detect radial velocity variations indicative of binarity in $6 \%$ of SDSS stars with repeated spectroscopic observations. Corrected for the sparse sampling of the study (most objects were only observed twice), Pourbaix et al. (2005) derive a total spectroscopic binary fraction of $18 \%$, consistent with the findings of a similar study of halo stars by Carney et al. (2003). This slightly overestimates the impact binaries could have in producing systems with odd colors, however, as the colors of binaries with large luminosity ratios are dominated by the primary, while binaries with more closely matched luminosities will tend to have similar colors. Binary systems with a non-mainsequence component, such as the WDMD pairs identified by Smolčić et al. (2004) and Silvestri et al. (2006) are a notable exception to this generalization, and are visible in the $u-g$ versus $g-r$ and $g-r$ versus $r-i$ color-color diagrams in Figure 2. Fortunately, such systems make up less than $0.1 \%$ of all stars detected in SDSS photometry (Smolčić et al. 2004), and so do not represent a serious source of contamination and bias for the median stellar locus measured here.

\subsection{Colors as a Function of Spectral Type}

To provide guidance in interpreting the properties of stars within the SDSS/2MASS stellar locus, we have estimated the
SDSS/2MASS colors of stars as a function of MK spectral type. We have used two independent methods: calculating synthetic SDSS/2MASS colors using flux-calibrated spectral standards, and assigning spectral types to stars with SDSS spectra and native SDSS photometry. We describe each of these methods in turn below.

\subsubsection{Synthetic SDSS/2MASS Photometry of Spectral Standards}

Pickles (1998) assembled a grid of flux calibrated spectral type standards with wavelength coverage extending from $1150 \AA$ to $2.5 \mu \mathrm{m}$ for solar metallicity stars and from 1150 to $10620 \AA$ for a selection of nonsolar metallicity stars. We have calculated "observed" fluxes in janskys from each spectrum by convolving and integrating the flux transmitted through the SDSS and 2MASS filters, ${ }^{14}$ and integrating the transmitted flux in janskys.

SDSS magnitudes were calculated from optical fluxes using the survey zero-point flux density ( $3631 \mathrm{Jy}$ ) and the asinh softening parameter ( $\mathrm{b}$ coefficients) for each filter, and applying offsets of $0.036,-0.012,-0.01,-0.028$, and -0.04 to our ugriz magnitudes, respectively, to account for the difference between the SDSS system and a perfect AB system. ${ }^{15}$ 2MASS magnitudes were calculated from near-infrared fluxes using Vega-based zero points of 1594,1024 , and $666.7 \mathrm{Jy}$ in the $J, H$, and $K_{s}$ filters, respectively (Cohen et al. 2003). Synthetic SDSS/2MASS colors for the solarmetallicity Pickles standards are presented in Tables 3 and 4, and shown in Figures 4 and 5; SDSS colors for the nonsolarmetallicity Pickles standards are given in Table 5, as the reduced spectral coverage prevents the calculation of synthetic 2MASS magnitudes for these stars. With uncertainties of a few percent in the absolute flux calibration of the spectra and the survey zero points, the synthetic colors presented in Tables 3 and 4 have characteristic uncertainties of $0.05 \mathrm{mag}$; as the Pickles spectra have only smoothed flux distributions in the near-infrared, the nearinfrared colors of these stars may be somewhat less accurate than the optical colors.

For completeness, we include in Table 3 absolute magnitudes calculated by Pickles (1998) for each spectrum, transformed onto the 2MASS system via the relations derived by Carpenter (2001) and reported as $M_{J}$ instead of $M_{K}$. We believe these approximate absolute magnitudes may be useful for back of the envelope calculations, particularly for giants and early-type (OBA) dwarfs. More reliable measurements of absolute magnitude as a function of SDSS/2MASS color exist for FGKM dwarf stars; for those stars, Williams et al. (2002), Hawley et al. (2002), West et al.

\footnotetext{
${ }^{14}$ SDSS and 2MASS filter curves, including the effects of atmospheric transmission, are available at http://www.sdss.org/dr5/instruments/imager/filters/ and http://spider.ipac.caltech.edu/staff/waw/2mass/opt_cal/index.html.

${ }^{15} \mathrm{See} \mathrm{http://www.sdss.org/dr5/algorithms/fluxcal.html} \mathrm{for} \mathrm{a} \mathrm{detailed} \mathrm{description}$ of the flux calibration of SDSS photometry, and http://cosmo.nyu.edu/blanton/ kcorrect/ for a description of the SDSS to $\mathrm{AB}$ conversion. Note, however, that the $\mathrm{AB}$ offsets assumed here differ slightly from those adopted by Eisenstein et al. (2006).
} 
TABLE 3

Synthetic SDSS/2MASS Photometry of Pickles (1998) Solar Metallicity Standards

\begin{tabular}{|c|c|c|c|c|c|c|c|c|c|}
\hline Spec. Type & Lum. Class & $u-g$ & $g-r$ & $r-i$ & $i-z$ & $z-J$ & $J-H$ & $H-K_{s}$ & $M_{J}$ \\
\hline 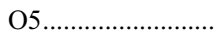 & $\mathrm{V}$ & -0.39 & -0.62 & -0.37 & -0.35 & 0.20 & -0.23 & -0.13 & -4.50 \\
\hline 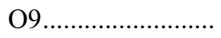 & $\mathrm{V}$ & -0.32 & -0.62 & -0.35 & -0.36 & 0.24 & -0.16 & -0.11 & -3.81 \\
\hline 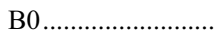 & $\mathrm{V}$ & -0.25 & -0.59 & -0.35 & -0.32 & 0.19 & -0.11 & -0.04 & -2.80 \\
\hline B1 .......................... & $\mathrm{V}$ & -0.13 & -0.49 & -0.33 & -0.26 & 0.13 & -0.06 & -0.03 & -2.03 \\
\hline $\mathrm{B} 3 \ldots . .$. & $\mathrm{V}$ & 0.18 & -0.46 & -0.26 & -0.26 & 0.20 & -0.22 & -0.08 & -1.55 \\
\hline B5/7 ................... & $\mathrm{V}$ & 0.51 & -0.39 & -0.23 & -0.23 & 0.27 & -0.01 & -0.02 & -1.37 \\
\hline 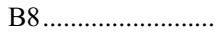 & $\mathrm{V}$ & 0.75 & -0.33 & -0.23 & -0.17 & 0.28 & 0.02 & 0.02 & -1.21 \\
\hline 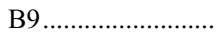 & $\mathrm{V}$ & 0.86 & -0.28 & -0.23 & -0.18 & 0.36 & 0.03 & -0.01 & -0.53 \\
\hline $\mathrm{A} 0 \ldots \ldots \ldots \ldots \ldots \ldots$ & $\mathrm{V}$ & 1.09 & -0.25 & -0.18 & -0.17 & 0.46 & 0.02 & -0.03 & 0.43 \\
\hline $\mathrm{A} 2 \ldots \ldots$ & $\mathrm{V}$ & 1.16 & -0.23 & -0.17 & -0.15 & 0.38 & -0.10 & -0.01 & 1.17 \\
\hline 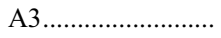 & $\mathrm{V}$ & 1.15 & -0.16 & -0.15 & -0.15 & 0.39 & -0.14 & -0.02 & 1.25 \\
\hline A5 ....................... & $\mathrm{V}$ & 1.20 & -0.10 & -0.11 & -0.10 & 0.55 & 0.09 & 0.01 & 1.38 \\
\hline 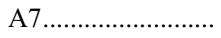 & $\mathrm{V}$ & 1.21 & -0.02 & -0.08 & -0.08 & 0.53 & 0.13 & 0.03 & 1.73 \\
\hline 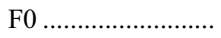 & $\mathrm{V}$ & 1.16 & 0.10 & -0.01 & -0.09 & 0.59 & 0.16 & 0.02 & 2.43 \\
\hline 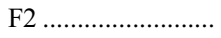 & $\mathrm{V}$ & 1.12 & 0.19 & 0.03 & -0.02 & 0.52 & 0.04 & -0.00 & 2.63 \\
\hline F5 & $\mathrm{V}$ & 1.09 & 0.26 & 0.03 & -0.02 & 0.71 & 0.22 & 0.00 & 2.62 \\
\hline 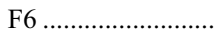 & $\mathrm{V}$ & 1.14 & 0.28 & 0.08 & -0.01 & 0.72 & 0.23 & 0.01 & 2.90 \\
\hline F8 ........... & $\mathrm{V}$ & 1.22 & 0.36 & 0.10 & 0.03 & 0.74 & 0.23 & 0.00 & 2.98 \\
\hline 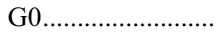 & $\mathrm{V}$ & 1.30 & 0.38 & 0.14 & 0.02 & 0.71 & 0.28 & 0.00 & 3.18 \\
\hline $\mathrm{G} 2 \ldots \ldots \ldots \ldots \ldots \ldots \ldots$ & $\mathrm{V}$ & 1.37 & 0.45 & 0.16 & 0.04 & 0.84 & 0.35 & 0.00 & 3.45 \\
\hline 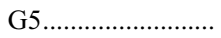 & $\mathrm{V}$ & 1.49 & 0.49 & 0.16 & 0.06 & 0.78 & 0.29 & 0.03 & 3.54 \\
\hline 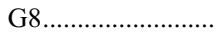 & $\mathrm{V}$ & 1.60 & 0.57 & 0.19 & 0.06 & 0.87 & 0.37 & 0.04 & 3.85 \\
\hline $\mathrm{K} 0 \ldots \ldots \ldots \ldots \ldots \ldots \ldots$ & $\mathrm{V}$ & 1.68 & 0.62 & 0.22 & 0.07 & 0.84 & 0.47 & 0.07 & 4.17 \\
\hline $\mathrm{K} 2 \ldots \ldots \ldots \ldots \ldots \ldots \ldots$ & $\mathrm{V}$ & 1.95 & 0.78 & 0.24 & 0.11 & 0.99 & 0.51 & 0.07 & 4.50 \\
\hline $\mathrm{K} 3 \ldots \ldots \ldots \ldots \ldots \ldots \ldots$ & $\mathrm{V}$ & 2.10 & 0.85 & 0.32 & 0.13 & 0.99 & 0.58 & 0.01 & 4.94 \\
\hline $\mathrm{K} 4 \ldots \ldots \ldots \ldots \ldots \ldots \ldots \ldots \ldots \ldots$ & $\mathrm{V}$ & 2.21 & 1.00 & 0.38 & 0.16 & 1.01 & 0.59 & 0.10 & 5.21 \\
\hline $\mathrm{K} 5 \ldots \ldots \ldots \ldots \ldots \ldots \ldots$ & $\mathrm{V}$ & 2.54 & 1.18 & 0.40 & 0.20 & 1.06 & 0.62 & 0.10 & 5.45 \\
\hline K7 ......................... & $\mathrm{V}$ & 2.56 & 1.34 & 0.54 & 0.30 & 1.01 & 0.60 & 0.11 & 5.77 \\
\hline 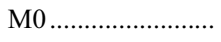 & $\mathrm{V}$ & 2.65 & 1.31 & 0.64 & 0.38 & 1.29 & 0.68 & 0.15 & 5.72 \\
\hline M1 ...................... & $\mathrm{V}$ & 2.72 & 1.33 & 0.78 & 0.46 & 1.20 & 0.67 & 0.25 & 6.04 \\
\hline M2 ....................... & $\mathrm{V}$ & 2.59 & 1.43 & 0.85 & 0.52 & 1.33 & 0.69 & 0.15 & 6.23 \\
\hline M2.5 .................... & $\mathrm{V}$ & 2.74 & 1.47 & 1.01 & 0.63 & 1.32 & 0.66 & 0.18 & 6.51 \\
\hline 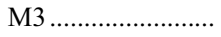 & $\mathrm{V}$ & 2.67 & 1.46 & 1.20 & 0.67 & 1.37 & 0.65 & 0.20 & 6.77 \\
\hline M4 ....................... & $\mathrm{V}$ & 3.13 & 1.48 & 1.51 & 0.76 & 1.56 & 0.63 & 0.23 & 7.06 \\
\hline 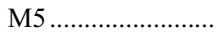 & $\mathrm{V}$ & 3.05 & 1.59 & 1.73 & 0.94 & 1.96 & 0.86 & 0.24 & 7.39 \\
\hline M6 ......................... & $\mathrm{V}$ & 2.99 & 1.72 & 2.12 & 1.24 & 2.31 & 0.66 & 0.32 & 7.72 \\
\hline 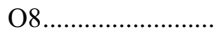 & III & -0.35 & -0.58 & -0.38 & -0.36 & 0.12 & 0.04 & -0.07 & -4.67 \\
\hline $\mathrm{B} 1 / 2 \ldots \ldots \ldots \ldots \ldots$ & III & -0.14 & -0.53 & -0.32 & -0.28 & 0.27 & -0.08 & -0.13 & -3.65 \\
\hline 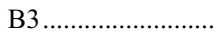 & III & 0.18 & -0.48 & -0.25 & -0.25 & 0.17 & 0.04 & 0.00 & -2.71 \\
\hline B5 & III & 0.42 & -0.42 & -0.23 & -0.24 & 0.29 & -0.06 & -0.01 & -2.16 \\
\hline В9 & III & 0.93 & -0.33 & -0.23 & -0.17 & 0.39 & -0.05 & 0.01 & -0.76 \\
\hline $\mathrm{A} 0 \ldots \ldots \ldots \ldots \ldots$ & III & 1.07 & -0.22 & -0.20 & -0.16 & 0.42 & 0.04 & 0.00 & -0.08 \\
\hline 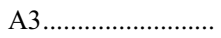 & III & 1.24 & -0.12 & -0.17 & -0.15 & 0.45 & -0.11 & -0.05 & 0.10 \\
\hline 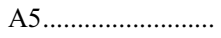 & III & 1.23 & -0.08 & -0.10 & -0.10 & 0.53 & 0.13 & -0.01 & 0.17 \\
\hline 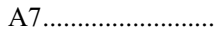 & III & 1.27 & -0.01 & -0.09 & -0.08 & 0.56 & 0.00 & -0.02 & 0.51 \\
\hline 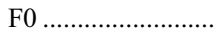 & III & 1.27 & 0.05 & -0.05 & -0.02 & 0.54 & 0.23 & 0.00 & 0.56 \\
\hline 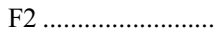 & III & 1.18 & 0.23 & 0.01 & -0.03 & 0.62 & 0.13 & -0.01 & 0.85 \\
\hline F5 ......................... & III & 1.21 & 0.24 & 0.05 & -0.02 & 0.67 & 0.33 & 0.00 & 0.83 \\
\hline G0 & III & 1.49 & 0.48 & 0.15 & 0.05 & 0.92 & 0.46 & -0.00 & 0.78 \\
\hline G5 ....................... & III & 1.86 & 0.68 & 0.22 & 0.08 & 0.99 & 0.48 & 0.03 & 1.82 \\
\hline 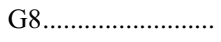 & III & 2.02 & 0.75 & 0.24 & 0.11 & 0.95 & 0.51 & 0.04 & 1.72 \\
\hline $\mathrm{K} 0 \ldots \ldots \ldots \ldots \ldots \ldots \ldots \ldots \ldots \ldots \ldots$ & III & 2.20 & 0.75 & 0.27 & 0.12 & 1.01 & 0.53 & 0.05 & 1.67 \\
\hline $\mathrm{K} 1 \ldots \ldots \ldots \ldots \ldots \ldots$ & III & 2.34 & 0.85 & 0.29 & 0.15 & 1.05 & 0.54 & 0.06 & 0.31 \\
\hline $\mathrm{K} 2 \ldots \ldots$ & III & 2.48 & 0.93 & 0.34 & 0.18 & 1.10 & 0.67 & 0.04 & -0.63 \\
\hline $\mathrm{K} 3 \ldots \ldots \ldots \ldots \ldots \ldots \ldots \ldots \ldots \ldots \ldots$ & III & 2.71 & 1.02 & 0.35 & 0.18 & 1.31 & 0.74 & 0.16 & -1.13 \\
\hline $\mathrm{K} 4 \ldots \ldots \ldots \ldots \ldots \ldots \ldots$ & III & 3.10 & 1.23 & 0.43 & 0.27 & 1.28 & 0.81 & 0.16 & -2.17 \\
\hline 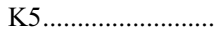 & III & 3.23 & 1.27 & 0.56 & 0.34 & 1.29 & 0.75 & 0.17 & -3.01 \\
\hline 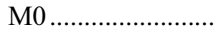 & III & 3.17 & 1.28 & 0.65 & 0.38 & 1.29 & 0.90 & 0.13 & -3.49 \\
\hline M1 ...................... & III & 3.16 & 1.29 & 0.73 & 0.43 & 1.32 & 0.92 & 0.12 & -3.71 \\
\hline 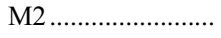 & III & 3.19 & 1.32 & 0.82 & 0.47 & 1.35 & 0.94 & 0.18 & -3.92 \\
\hline M3 ....................... & III & 3.13 & 1.27 & 1.01 & 0.58 & 1.38 & 0.95 & 0.17 & -4.15 \\
\hline M4 .......................... & III & 3.01 & 1.22 & 1.29 & 0.73 & 1.49 & 0.95 & 0.20 & -4.51 \\
\hline 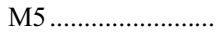 & III & 2.68 & 1.13 & 1.64 & 0.93 & 1.68 & 0.99 & 0.22 & -4.75 \\
\hline 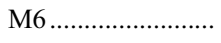 & III & 2.43 & 1.11 & 1.99 & 1.18 & 1.94 & 1.05 & 0.22 & -5.28 \\
\hline M7 .......................... & III & 1.82 & 1.15 & 2.36 & 1.58 & 2.29 & 1.08 & 0.22 & -5.64 \\
\hline
\end{tabular}


TABLE 3-Continued

\begin{tabular}{|c|c|c|c|c|c|c|c|c|c|}
\hline Spec. Type & Lum. Class & $u-g$ & $g-r$ & $r-i$ & $i-z$ & $z-J$ & $J-H$ & $H-K_{s}$ & $M_{J}$ \\
\hline M8 .......................... & III & 1.33 & 1.33 & 2.45 & 2.03 & 2.93 & 1.07 & 0.26 & -6.13 \\
\hline M9 ............ & III & 1.24 & 1.16 & 2.37 & 2.01 & 3.17 & 0.97 & 0.3 & -6.60 \\
\hline 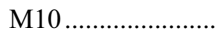 & III & 1.12 & 0.89 & 2.58 & 2.22 & 3.22 & 0.83 & 0.4 & -7.04 \\
\hline 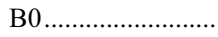 & I & -0.23 & -0.50 & -0.28 & -0.31 & 0.22 & -0.02 & -0.13 & -6.59 \\
\hline B1 ............................ & I & -0.14 & -0.42 & -0.26 & -0.34 & 0.27 & -0.09 & -0.09 & -6.23 \\
\hline 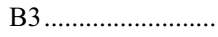 & I & 0.08 & -0.36 & -0.27 & -0.28 & 0.37 & 0.00 & 0.01 & -6.28 \\
\hline 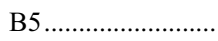 & I & 0.17 & -0.30 & -0.18 & -0.19 & 0.32 & 0.04 & -0.01 & -6.19 \\
\hline 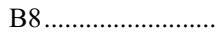 & I & 0.37 & -0.27 & -0.17 & -0.13 & 0.37 & -0.03 & 0.03 & -6.64 \\
\hline A0 .......................... & I & 0.81 & -0.23 & -0.16 & -0.10 & 0.44 & 0.12 & -0.02 & -6.62 \\
\hline A2 & $\mathrm{I}$ & 0.83 & -0.11 & -0.14 & -0.13 & 0.51 & 0.15 & -0.02 & -6.87 \\
\hline 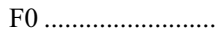 & I & 1.47 & -0.02 & -0.03 & -0.03 & 0.50 & 0.15 & -0.01 & -7.17 \\
\hline F5 & I & 1.43 & 0.06 & -0.00 & -0.03 & 0.65 & 0.13 & -0.02 & -7.37 \\
\hline F8 & I & 1.72 & 0.37 & 0.03 & -0.03 & 0.73 & 0.30 & 0.06 & -7.56 \\
\hline 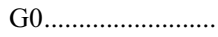 & I & 1.73 & 0.58 & 0.10 & -0.02 & 0.74 & 0.35 & 0.08 & -7.67 \\
\hline G5 & $\mathrm{I}$ & 2.22 & 0.82 & 0.17 & 0.03 & 0.84 & 0.42 & 0.09 & -7.74 \\
\hline G8 & I & 2.65 & 1.00 & 0.27 & 0.04 & 0.78 & 0.33 & 0.03 & -7.82 \\
\hline $\mathrm{K} 2 \ldots \ldots \ldots \ldots \ldots \ldots \ldots \ldots \ldots \ldots \ldots \ldots \ldots$ & $\mathrm{I}$ & 3.21 & 1.27 & 0.40 & 0.17 & 0.82 & 0.37 & 0.08 & -8.06 \\
\hline 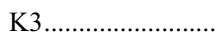 & $\mathrm{I}$ & 3.30 & 1.38 & 0.47 & 0.17 & 1.03 & 0.58 & 0.08 & -8.17 \\
\hline 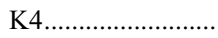 & I & 3.30 & 1.47 & 0.60 & 0.38 & 1.23 & 0.74 & 0.21 & -8.40 \\
\hline M2 ........................ & $\mathrm{I}$ & 3.51 & 1.57 & 1.05 & 0.55 & 1.10 & 0.69 & 0.34 & -10.04 \\
\hline
\end{tabular}

Notes.-Table 3 is also available in machine-readable form in the electronic edition of the Astronomical Journal.

TABLE 4

$g-i$ as a Function of MK Spectral Type

\begin{tabular}{|c|c|c|c|}
\hline Spec. Type & Synth. $g-i$ & Spec. $g-i$ & $n_{\text {spec }}$ \\
\hline$\ldots \ldots \ldots$ & -1.00 & $\ldots$ & 0 \\
\hline 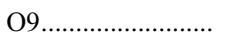 & -0.97 & $\ldots$ & 0 \\
\hline 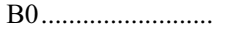 & -0.94 & $\ldots$ & 0 \\
\hline 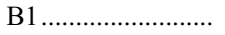 & -0.82 & $\ldots$ & 0 \\
\hline В3 …….................. & -0.72 & $\ldots$ & 0 \\
\hline 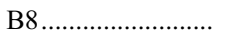 & -0.57 & $\ldots$ & 0 \\
\hline 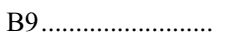 & -0.51 & $\ldots$ & 0 \\
\hline $\mathrm{A} 0$ & -0.44 & $\ldots$ & 0 \\
\hline $\mathrm{A} 2 \ldots \ldots$ & -0.39 & $\ldots$ & 0 \\
\hline A $3 \ldots \ldots \ldots \ldots$ & -0.30 & $\ldots$ & 0 \\
\hline A5 & -0.21 & $\ldots$ & 0 \\
\hline 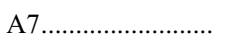 & -0.10 & $\ldots$ & 0 \\
\hline 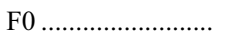 & 0.09 & 0.41 & 1 \\
\hline F2 . & 0.22 & 0.40 & 24 \\
\hline 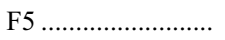 & 0.29 & 0.46 & 16 \\
\hline 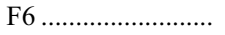 & 0.36 & 0.46 & 33 \\
\hline F8 ……....................... & 0.45 & 0.55 & 97 \\
\hline 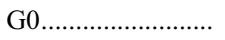 & 0.52 & 0.57 & 53 \\
\hline 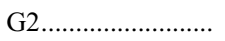 & 0.60 & 0.60 & 49 \\
\hline 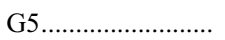 & 0.65 & 0.66 & 58 \\
\hline 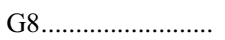 & 0.76 & 0.77 & 95 \\
\hline $\mathrm{K} 0 \ldots \ldots \ldots \ldots \ldots \ldots \ldots \ldots$ & 0.83 & 0.81 & 80 \\
\hline $\mathrm{K} 2 \ldots \ldots \ldots \ldots \ldots \ldots \ldots$ & 1.02 & 0.95 & 275 \\
\hline $\mathrm{K} 3 \ldots \ldots \ldots \ldots \ldots \ldots$ & 1.17 & 1.10 & 88 \\
\hline 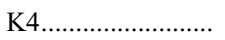 & 1.38 & 1.31 & 274 \\
\hline 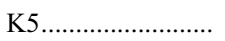 & 1.59 & 1.47 & 137 \\
\hline 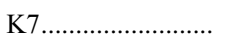 & 1.88 & 1.75 & 148 \\
\hline 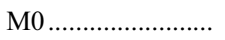 & 1.95 & 1.98 & 315 \\
\hline M1 ………............. & 2.10 & 2.23 & 256 \\
\hline 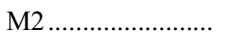 & 2.28 & 2.42 & 303 \\
\hline 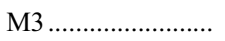 & 2.66 & 2.63 & 438 \\
\hline 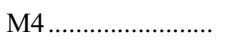 & 2.99 & 2.87 & 255 \\
\hline 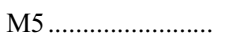 & 3.32 & 3.22 & 57 \\
\hline 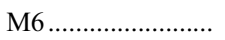 & 3.84 & 3.39 & 13 \\
\hline
\end{tabular}

(2005), Bilir et al. (2005), Davenport et al. (2006), Bochanski et al. (2007), and D. A. Golimowski et al. (2008, in preparation) present science-grade empirical measurements, while Girardi et al. (2004) and Dotter et al. (2007) provide stellar models with SDSS photometry.

\subsubsection{Spectral Typing SDSS Stars}

To provide a check on the accuracy of the synthetic colors described above, we have assigned spectral types to stars with SDSS spectroscopy and native SDSS photometry. These types were assigned using a custom IDL package, dubbed "the Hammer," whose algorithm is described in full in Appendix A. In short, the Hammer automatically assigns spectral types to input spectra by measuring a suite of spectral indices and performing a leastsquares minimization of the residuals between the indices of the target and those measured from spectral type standards. This code has been made available for community use, with an archival version available from the Astronomical Journal in the electronic edition of this article. Updated versions of the Hammer can also be downloaded from the Hammer's Web site. ${ }^{16}$

To measure the median SDSS colors of the MK subclasses, we used the Hammer to assign MK spectral types to 3443 SDSS stellar spectra. Input SDSS spectra were selected from a subset of SDSS plates that sparsely sample the SDSS color space inhabited by point sources. ${ }^{17}$ Mean fluxes and S/Ns were then calculated in three $100 \AA$ bands centered at 4550, 6150, and $8300 \AA$; if the wavelength band with the largest mean flux had $\mathrm{S} / \mathrm{N}<10$ per wavelength element, the spectrum was rejected as too noisy for further analysis. These spectra were classified automatically, and all types were visually confirmed by KRC. The colors of these stars are shown in Figures 4 and 5 as a function of their assigned spectral type, with the associated $g-i$ versus spectral

\footnotetext{
${ }^{16}$ See http://www.cfa.harvard.edu/ kcovey/thehammer.

17 This sample is known as the "Spectra of Everything" sample, contained in part on plates $1062-1075,1077-1088,1090-1096,1101,1103-1107,1116-1117$, 1473-1476, 1487-1488, 1492-1497, 1504-1505, 1508-1509, 1511, 1514$1518,1521-1523$, and 1529 , and was originally targeted to test the completeness of the quasar spectroscopic targeting algorithm (Richards et al. 2002).
} 

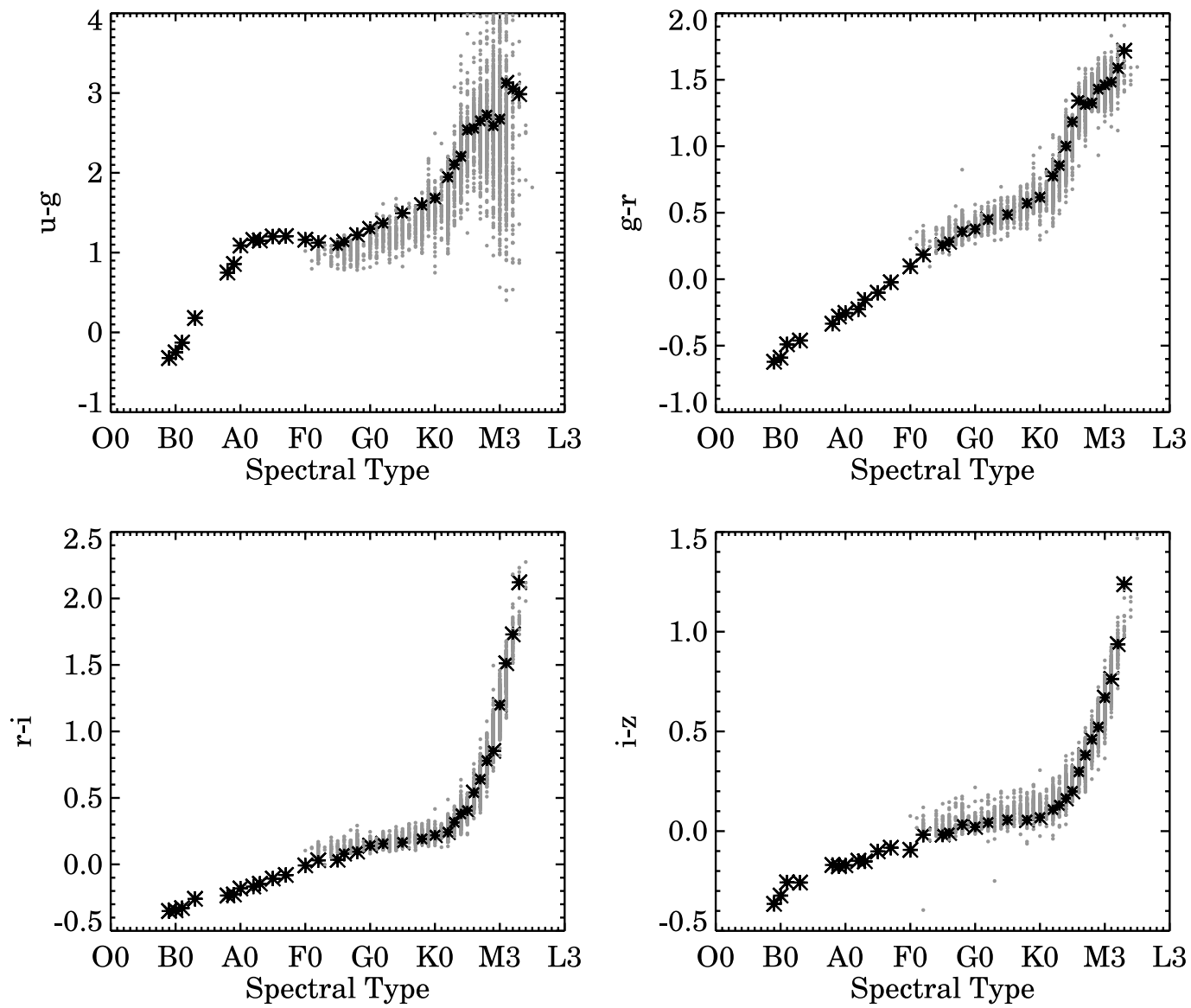

FIG. 4.-Relationship between stellar MK spectral type and color in the SDSS photometric system. Black asterisks show synthetic SDSS colors calculated for the Pickles (1998) spectral atlas, and gray dots show the colors of 3443 stars with spectral types assigned to SDSS spectra using the Hammer spectral typing software.

type relationship presented in Table 4 . Note that this sample contained no stars bluer than $g-i=0.0$; equivalently, no stars were assigned spectral types earlier than F0.

\subsubsection{Comparing the Color-Spectral Type Relations}

The color-spectral type relations obtained from native and synthetic SDSS photometry agree well, particularly for the $g-r$ and

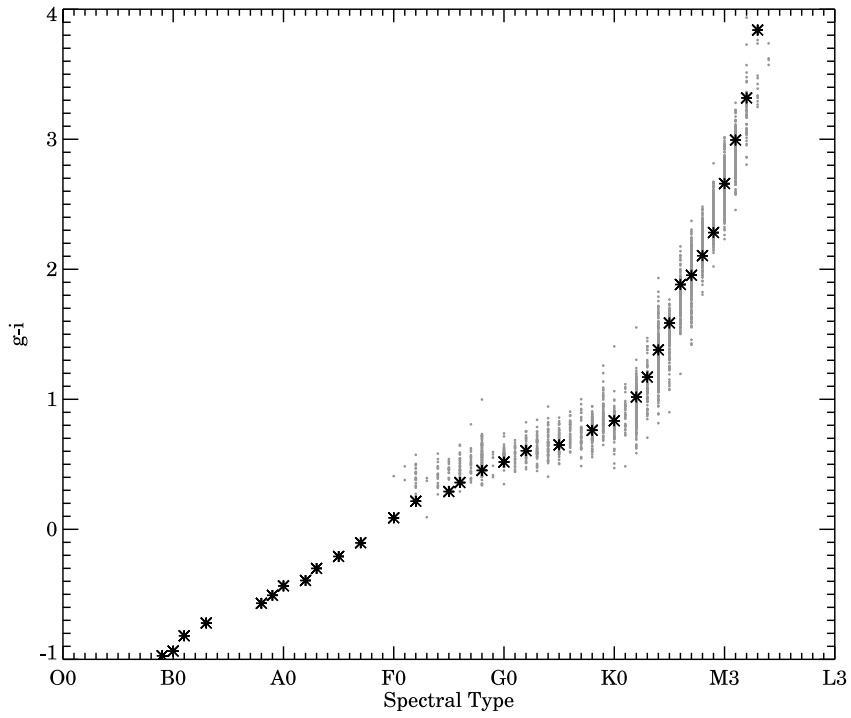

FIG. 5.-Relationship between stellar MK spectral type and $g-i$ color in the SDSS photometric system. Symbols are the same as in Fig. 4.
TABLE 5

Synthetic SDSS Photometry of Pickles (1998) Non-Solar Metallicity Standards

\begin{tabular}{|c|c|c|c|c|c|c|}
\hline Spec. Type & Lum. Class & $u-g$ & $g-r$ & $r-i$ & $i-z$ & {$[\mathrm{Fe} / \mathrm{H}]$} \\
\hline wF5 …............... & V & 1.03 & 0.26 & 0.05 & -0.04 & -0.30 \\
\hline rF6 .................... & $\mathrm{V}$ & 1.20 & 0.33 & 0.10 & 0.00 & 0.30 \\
\hline wF8 ....... & V & 1.16 & 0.32 & 0.10 & -0.03 & -0.60 \\
\hline $\mathrm{rF} 8 \ldots . .$. & V & 1.27 & 0.38 & 0.15 & -0.01 & 0.20 \\
\hline wG0... & V & 1.28 & 0.37 & 0.13 & 0.03 & -0.80 \\
\hline rG0 ....... & V & 1.36 & 0.41 & 0.16 & 0.04 & 0.40 \\
\hline wG5 .................. & V & 1.30 & 0.47 & 0.13 & 0.03 & -0.40 \\
\hline 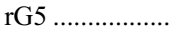 & V & 1.51 & 0.51 & 0.14 & 0.05 & 0.10 \\
\hline rK0 .................... & V & 1.83 & 0.67 & 0.19 & 0.09 & 0.50 \\
\hline wG5 .................. & III & 1.69 & 0.65 & 0.21 & 0.10 & -0.26 \\
\hline rG5 $\ldots \ldots \ldots \ldots \ldots \ldots$ & III & 1.85 & 0.69 & 0.22 & 0.08 & 0.23 \\
\hline 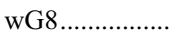 & III & 2.01 & 0.68 & 0.25 & 0.11 & -0.38 \\
\hline wK0 ................... & III & 1.92 & 0.75 & 0.26 & 0.11 & -0.33 \\
\hline rK0 ..... & III & 2.29 & 0.81 & 0.27 & 0.13 & 0.18 \\
\hline wK1 ................... & III & 2.20 & 0.82 & 0.27 & 0.13 & -0.10 \\
\hline rK1 .................. & III & 2.62 & 0.91 & 0.30 & 0.17 & 0.27 \\
\hline wK2 ...... & III & 2.46 & 0.84 & 0.32 & 0.15 & -0.38 \\
\hline rK2 …................. & III & 2.65 & 0.99 & 0.33 & 0.18 & 0.24 \\
\hline wK3 .................. & III & 2.47 & 0.96 & 0.37 & 0.19 & -0.36 \\
\hline rK3 …................ & III & 2.92 & 1.10 & 0.36 & 0.21 & 0.27 \\
\hline 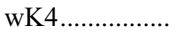 & III & 2.91 & 1.17 & 0.42 & 0.26 & -0.33 \\
\hline 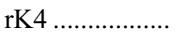 & III & 2.87 & 1.22 & 0.43 & 0.29 & 0.15 \\
\hline rK5 …................ & III & 3.34 & 1.35 & 0.56 & 0.32 & 0.08 \\
\hline
\end{tabular}


$r-i$ colors that underly the $g-i$ color used in $\S 3.2$ to parameterize the median stellar locus. Residuals between the SDSS colors of Pickles (1998) standards and SDSS stars assigned the same spectral type are typically 0.05 magnitudes in $g-r$ and $r-i$ (producing $g-i$ residuals of $\sim 0.1 \mathrm{mag}$ ), and slightly larger in $i-z(\sim 0.08 \mathrm{mag})$. The agreement in $u-g$ is worse, with native SDSS stars consistently bluer than the Pickles (1998) stars by 0.2 mags in color. This is easily understood as a metallicity effect; as $[\mathrm{Fe} / \mathrm{H}] \sim-0.75$ for typical SDSS stars, they suffer less line blanketing than the solar-metallicity Pickles standards, with accordingly bluer $u-g$ colors ( $\breve{Z}$. Ivezić et al. 2008, in preparation).

As the relationship between $g-i$ color and spectral type appears secure to within $0.1 \mathrm{mag}$ in color, Table 4 allows preliminary spectral types to be assigned to SDSS stars based on $g-i$ color alone. With $g-i$ color increasing by $\sim 0.05$ mag between spectral type subclasses, classification accuracies of $\pm 2-3$ subclasses should be achievable for stars with small photometric errors.

\section{CATALOGING COLOR OUTLIERS}

\subsection{Determining Color Distances}

Having characterized the adjacent colors of the stellar locus from 0.3 to $2.2 \mu \mathrm{m}$, we can now search for color outliers - objects whose colors place them in areas of color space well separated from the stellar locus. In calculating the statistical significance of an object's separation from the standard stellar locus, we account for photometric errors, as well as the intrinsic width of the stellar locus. This catalog will enable efficient and robust searches for color outliers by avoiding objects that live in sparsely populated areas of color-color space merely due to large photometric errors, and more interestingly, by aggregating the effects of small offsets in multiple colors. This algorithm resembles that used to select quasar candidates from SDSS imaging for spectroscopic observation, extended to account for an object's NIR colors (see Appendices A and B of Richards et al. 2002).

We calculate a quantity we dub the seven-dimensional color distance (7DCD), which captures the statistical significance of the distance in color space between an object (the "target") and a given "locus point" (characterized by colors from a single row in Table 1) as

$$
\text { 7DCD }=\sum_{k=0}^{6} \frac{\left(X_{k}^{\text {targ }}-X_{k}^{\text {locus }}\right)^{2}}{\sigma_{X}^{2}(\text { locus })+\sigma_{x}^{2}}
$$

where $X_{0}=u-g, X_{1}=g-r$, etc. In the above equation, the target's $X_{k}$ color error $\left(\sigma_{x}\right)$ is defined as the quadrature sum of the photometric errors reported in either the SDSS or 2MASS catalog in both appropriate filters. Normalizing the 7DCD by the target's photometric errors, as well as the observed width of the stellar locus, which itself includes the effects of photometric errors, would "double-count" the ability of photometric error to explain the separation of a source from the stellar locus; this effect was first detected by comparing preliminary 7DCD distributions to synthetic $\chi^{2}$-based distributions, which predicted 7DCD distributions larger than those actually observed. This was an especially troubling fact given that SDSS error estimates are, if anything, underestimating errors by $\sim 10 \%-20 \%$ (Scranton et al. 2005), which should lead to overestimates of the 7DCD of a given source.

We account for this effect by including only the intrinsic width of the stellar locus in the normalization of the color distance; $\sigma_{X}$

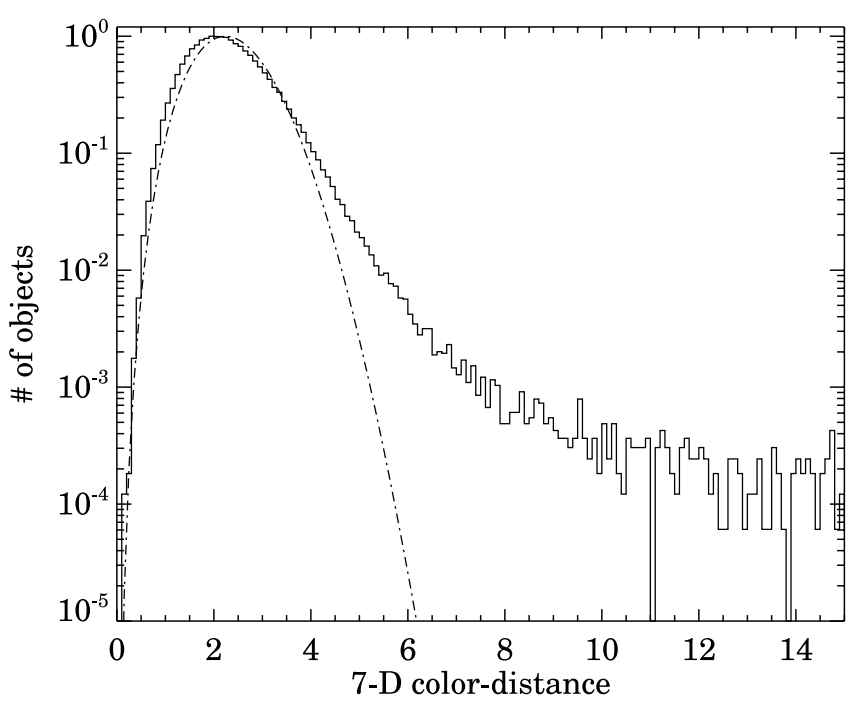

FIG. 6.- Seven-dimensional color distances for objects in the high-quality sample (solid line), compared to the $\chi^{2}$ distribution expected for a sample with 6 degrees of freedom (dash-dotted line). The sample contains a clear excess of sources with color distances $>6$.

gives the intrinsic width of the stellar locus in the $X_{k}$ color at the locus point, calculated by subtracting (in quadrature) the median color error from the standard deviation of the colors of objects in this $g-i$ bin in the high quality sample. The intrinsic width of the stellar locus therefore accounts for the range of colors induced by variations in stellar properties (e.g., metallicity; Ž. Ivezić et al. 2008 , in preparation), as well as instrumental spread not captured in the pipeline error estimates, such as that due to the red leak. It excludes, however, the component of the locus's width which is due purely to photometric errors that are well estimated by the SDSS photometric pipeline, and eliminates the double-counting of the importance of photometric errors in the calculation of the 7DCD.

Using this algorithm, and adopting minimum errors of 0.03 mag in the SDSS $u-g, g-r, r-i$, and $i-z$ colors, we have identified the minimum 7DCD and best-fit $g-i$ point along the median stellar locus for every object in our matched sample. Strictly speaking, the minimum 7DCD is not the minimum perpendicular distance between a given star and the stellar locus, which requires a continuous description of the stellar locus. As the polynomial fits given in $\S 3.2$ possess nontrivial residuals at extreme colors, we have chosen instead to calculate color distances using the discrete tabulation of the stellar locus given in Table 1. As Table 1 is finely spaced in $g-i$ color, the difference between the calculated color distance and the minimum perpendicular color distance will be small, particularly for objects with large color distances. This procedure also neglects the effects of photometric covariance, which Scranton et al. (2005) show can cause SDSS color errors to be underestimated by $\sim 20 \%$ for variable stars. Scranton et al. (2005) demonstrate, however, that the effect is negligible for nonvariable stars; since the majority of our sample is composed of nonvariable stars, we expect the effects of neglecting covariance to be small.

A histogram of the 7DCDs in our high-quality sample is shown in Figure 6, with the expected $\chi^{2}$ distribution shown for comparison. The $\chi^{2}$ distribution shown is calculated for a sample with 6 degrees of freedom, with 1 degree of freedom for each adjacent color and 1 degree of freedom removed to account for fitting the object to the closest $g-i$ bin. The observed distribution has a shallower slope than the expected distribution between color 

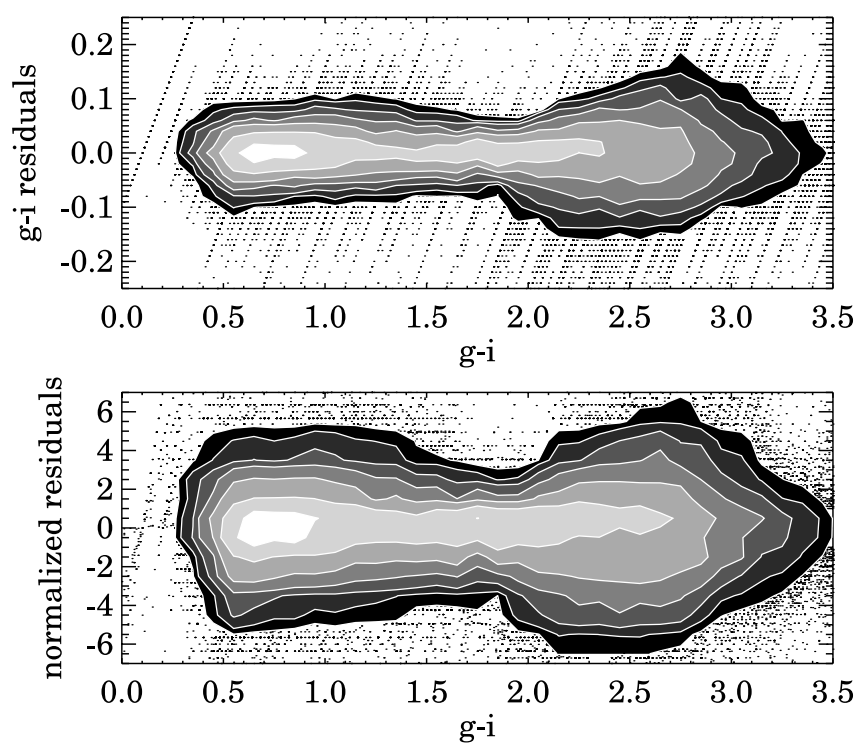

Fig. 7.- Residuals between the $g-i$ colors of the matched sample and the $g-i$ colors of the locus point that minimizes each source's 7DCD, shown as a function of $g-i$ color and before (top) and after (bottom) normalizing by each source's $g-i$ error. Successive contours indicate an increase in source density by a factor of 2 . More than $95 \%$ of the sample have $g-i$ residuals less than $0.1 \mathrm{mag}$, indicating that the position of normal stars within the SDSS/2MASS stellar locus can be accurately determined by minimizing their 7DCD.

distances of 3 and 6 -we attribute this to the intrinsic width of the stellar locus. Although equation (2) explicitly includes a term to account for the width of the stellar locus, it still implicitly assumes that the stellar locus can be modeled as a centrally concentrated Gaussian - if the stellar locus is significantly non-Gaussian, we would expect to see an excess of sources at large color distances.

The slope of 7DCDs changes at color distances larger than 6, becoming noticably shallower. This change in slope indicates that there are either true outliers whose distribution in color space differs from objects within the stellar locus (either for astrophysical reasons, or because of unrecognized photometric errors), or that our technique for assigning 7DCDs to individual objects is erroneously assigning high color distances to some subset of our catalog. In the following section we perform a variety of tests to understand the robustness, accuracy, and limitations of our derived 7DCDs.

\subsection{Understanding the Utility and Limitations of Color Distances}

In order to verify that the 7DCD provides an accurate means of identifying point-source color outliers, we have conducted a number of tests on the objects within our catalog. A set of tests to probe the sensitivity of the 7DCD to various source characteristics are shown in Figures 7-12. These investigations reveal the following properties of the 7DCDs calculated for the matched SDSS/2MASS sample:

1. Minimizing the 7DCD correctly identifies the location of normal stars along the median SDSS/2MASS stellar locus. As Figure 7 demonstrates, the $g-i$ color of the best-fit locus point matches a star's observed $g-i$ color within 0.1 magnitudes for more than $95 \%$ of the sample.

2. Stars with unusual colors are correctly assigned large 7DCDs; Figure 8 shows that stars in two example regions of unusual color space $\left(u-g<0.8, J-K_{s}>1.2\right)$ have median 7DCDs $>6$.

3 . The 7DCD is largely insensitive to the $i$ magnitude and $g-i$ color of a source. Figure 9 shows that brighter, bluer objects
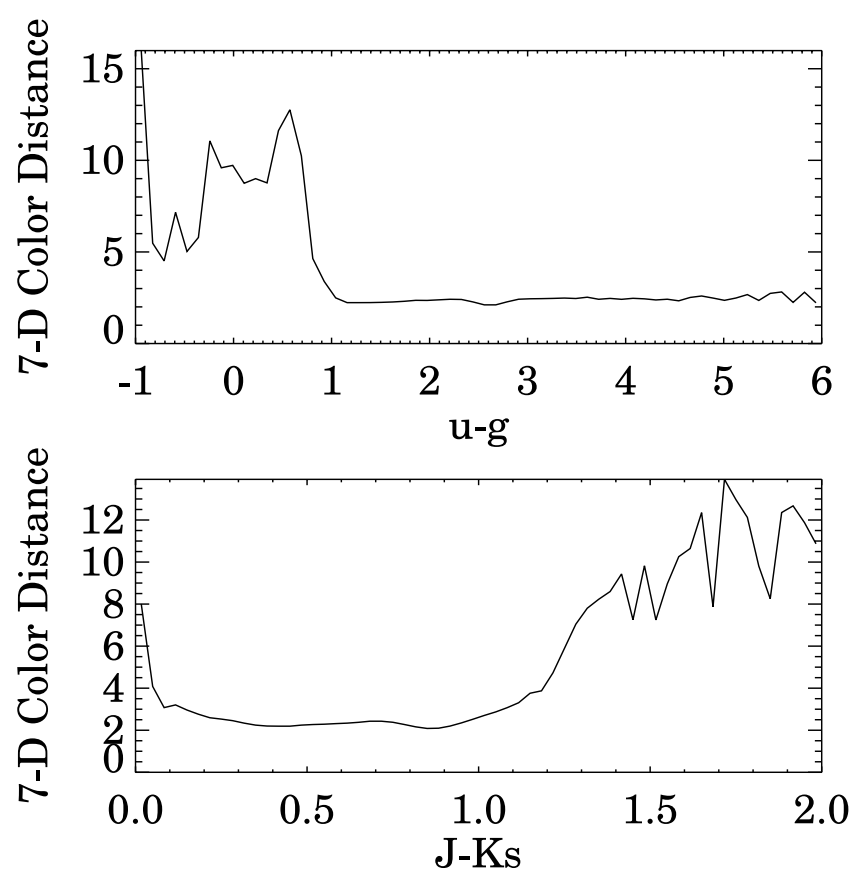

FIG. 8.-Median color distances for sources in bins of $u-g$ and $J-K_{s}$ color (top and bottom panels, respectively). Color distances increase sharply for $u-$ $g<1.0$ and $J-K_{s}>1.2$, which are examples of areas of color-space outside the SDSS/2MASS stellar locus.

slightly dominate the number of objects in a given color distance bin. This is consistent, however, with the larger overall fraction of bright, blue objects within our sample (see last panel of Fig. 2, where the peak stellar density occurs at $15<i<16$ and $g-i<1$ ). Aside from this effect, contours are essentially horizontal, implying that to first order color distances derived here are independent of magnitude and $g-i$ color.
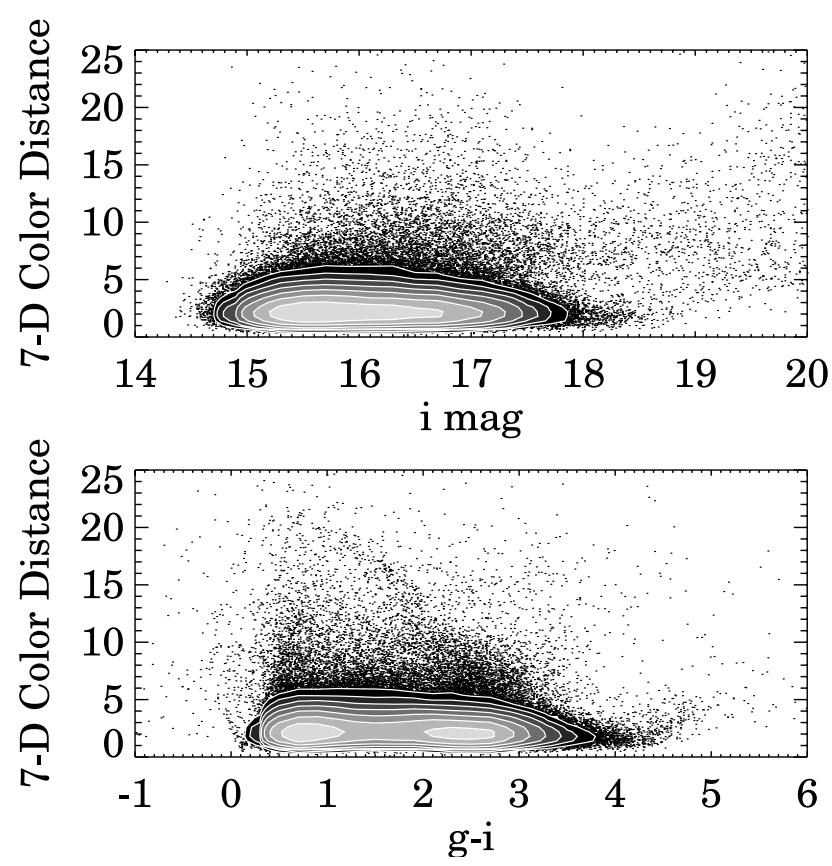

FIG. 9.- Seven-dimensional color distances calculated for the matched SDSS/ 2MASS sample, displayed as a function of $i$ magnitude (top) and $g-i$ color (bottom). Individual stars are shown as black dots, and contours indicate source density in areas too crowded for individual points to be distinguished from one another. Steps between contours indicate a doubling in source density. 


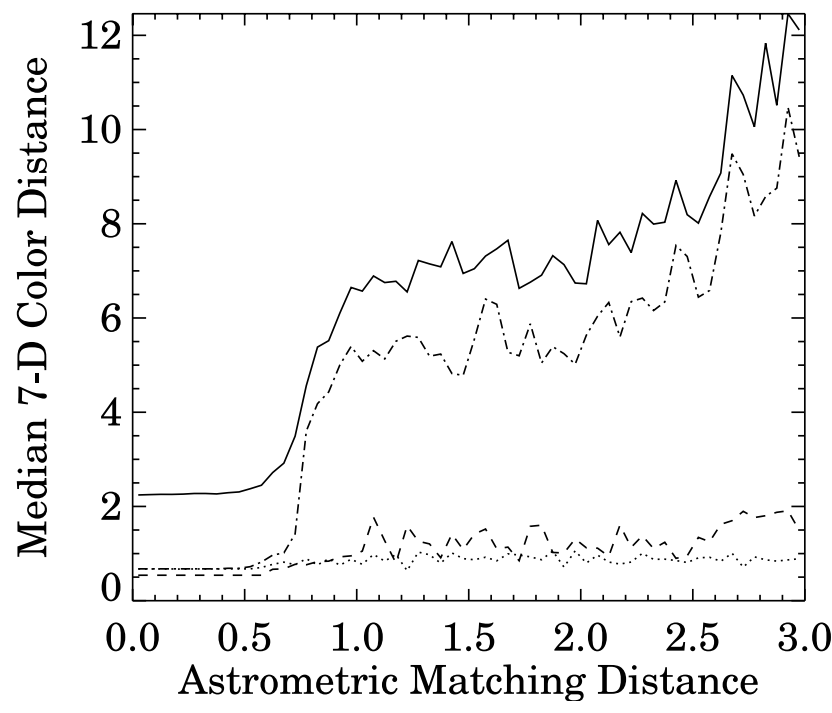

FIG. 10.-Median 7DCD (solid line) as a function of the astrometric distance between each matched object's SDSS and 2MASS detections. Plotted for comparison are the size of the $i-z$ (dashed line), $J-H$ (dotted line), and $z-J$ (dashdotted line) color-distance components, also as a function of astrometric distance. The $i-z$ and $J-H$ color distances are relatively insensitive to astrometric matching distance, as they depend only on an object's colors within a single survey, so are unaffected by incorrect matches between the two surveys. In contrast, the $z-J$ color distance increases sharply for objects whose SDSS and 2MASS positions are separated by more than $0.6^{\prime \prime}$, as distinct SDSS and 2MASS objects are increasingly spuriously identified as a single matched detection, producing abnormal $z-J$ colors.

4. Mismatches between the SDSS-2MASS catalogs generate a population of objects with spuriously high 7DCDs. This effect is visible in Figure 10, where derived color distances increase with astrometric matching distance (the distance between the positions of an object's SDSS and 2MASS counterparts) past $\sim 0.6^{\prime \prime}$.

Spurious associations generally occur between members of a visual binary, when the SDSS detection of the faint star is associated with the 2MASS detection of its brighter neighbor, resulting in anomalously red $z-J$ colors for the "matched" object.

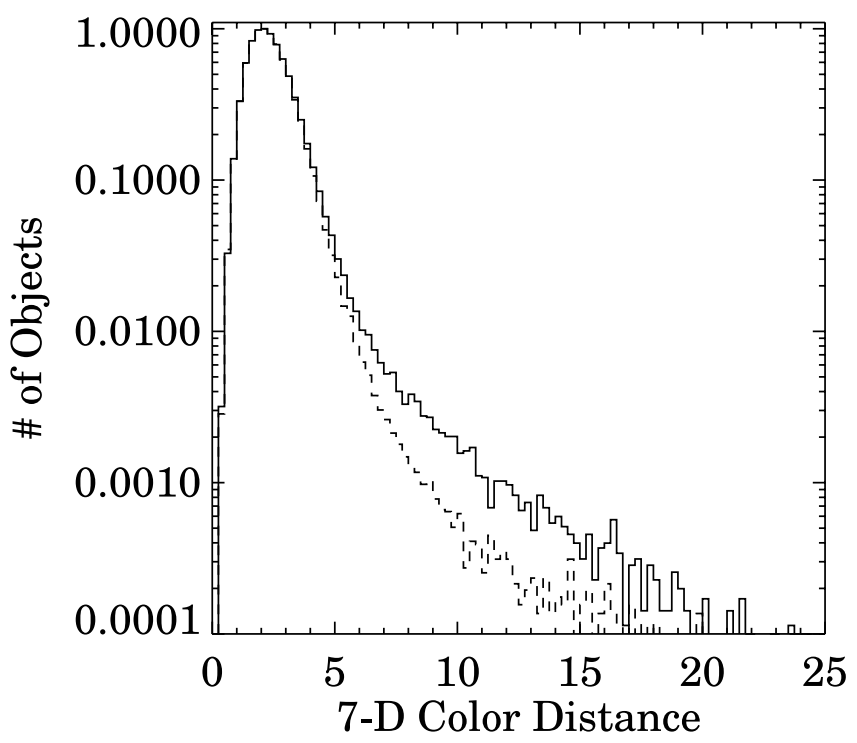

FIG. 11.-Seven-dimensional color distances calculated for objects with (solid line) and without (dashed line) the SDSS CHILD flag set. Although the two distributions match well for color distances less than 6 , deblended CHILDren produce a clear excess of color outliers at large color distances.

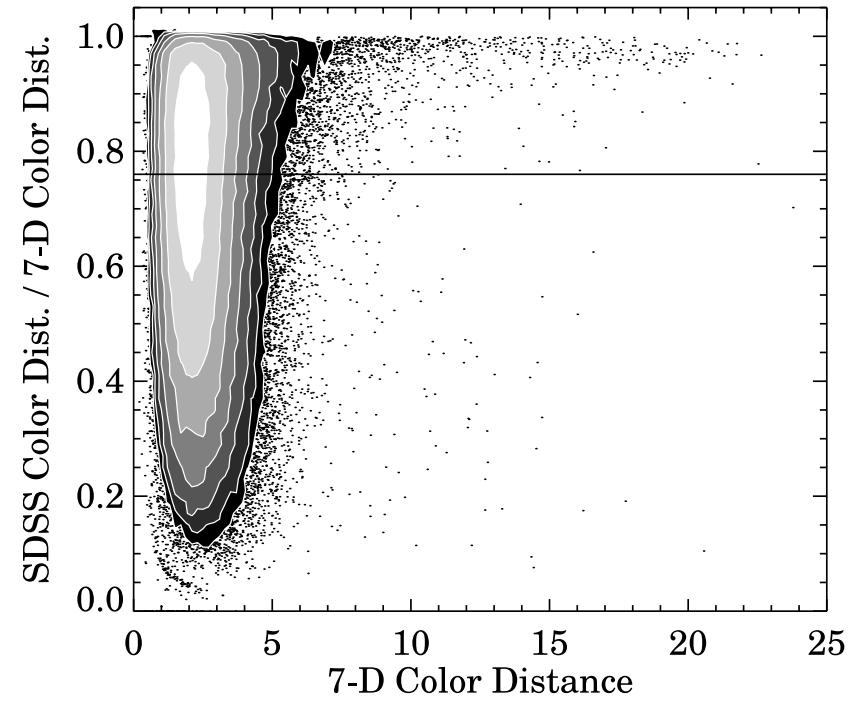

FIG. 12.- Ratio of SDSS-only to 7DCD as a function of 7DCD, calculated for objects with astrometric matching distances $<0.6^{\prime \prime}$ and CHILD $=0$. Steps between contours indicate a doubling of source densities. Shown for comparison, as a dashdotted line, is the 0.76 ratio expected if color offsets contribute equally from all filters. The SDSS-only : 7DCD ratio is nearly 1 for high-confidence color outliers (7DCDs $>6$ ), indicating that optical/SDSS colors provide the bulk of the leverage for identifying sources with unusual colors.

Given that SDSS filters make up five of the eight filters used to calculate the colors considered here, that the NIR colors of mainsequence stars change relatively slowly along the main sequence, and that SDSS photometry generally has smaller photometric errors than 2MASS for sources of similar magnitude, mismatches are typically fit to a $g-i$ bin along the stellar locus consistent with their SDSS colors. This results in relatively small residuals between the colors of the $g-i$ bin identified as the best fit for the object and the colors of that source in a single survey. The overwhelmingly red $z-J$ color, however, is a very poor match for the $z-J$ color of the object's best fit $g-i$ bin. This effect is visible in Figure 10, where the single-band $z-J$ color distance clearly increases for sources with astrometric matching distances $>0.6^{\prime \prime}$, with no similar increase detected in the $i-z$ or $J-H$ singlesurvey color distances.

Mismatches will be significant contaminants for any sample of sources with large 7DCDs; while sources with astrometric match distances greater than $0.6^{\prime \prime}$ make up only $0.75 \%$ of the SDSS/ 2 MASS sample, they make up $26 \%$ of the sources with 7DCD $>7$.

5. Objects whose SDSS counterpart is a deblended CHILD produce a disproportionate number of objects with large color distances, as seen in Figure 11; while CHILDren are only $41 \%$ of our sample, they make up 74\% of the sources with 7DCDs greater than 6. Although the astrometric position of a source may agree in both surveys to within $0.6^{\prime \prime}$, the SDSS deblended PSF photometry significantly reduces the amount of flux contributed by an object's nearby neighbor, while 2MASS's aperture photometry does not. This mismatch again produces sources with normal single survey colors and anomalously red $z-J$ colors, spuriously doubling the number of CHILDren assigned large color distances.

These tests indicate that 7DCD is a useful tool for identifying point sources in unusual areas of color space, and also enables the best-fit $g-i$ color to serve as a simple 1D parameterization of the properties of typical main-sequence stars. The most robust searches for color outliers using $7 D C D$ s, however, should be limited to sources with SDSS/2MASS astrometric matching distances 

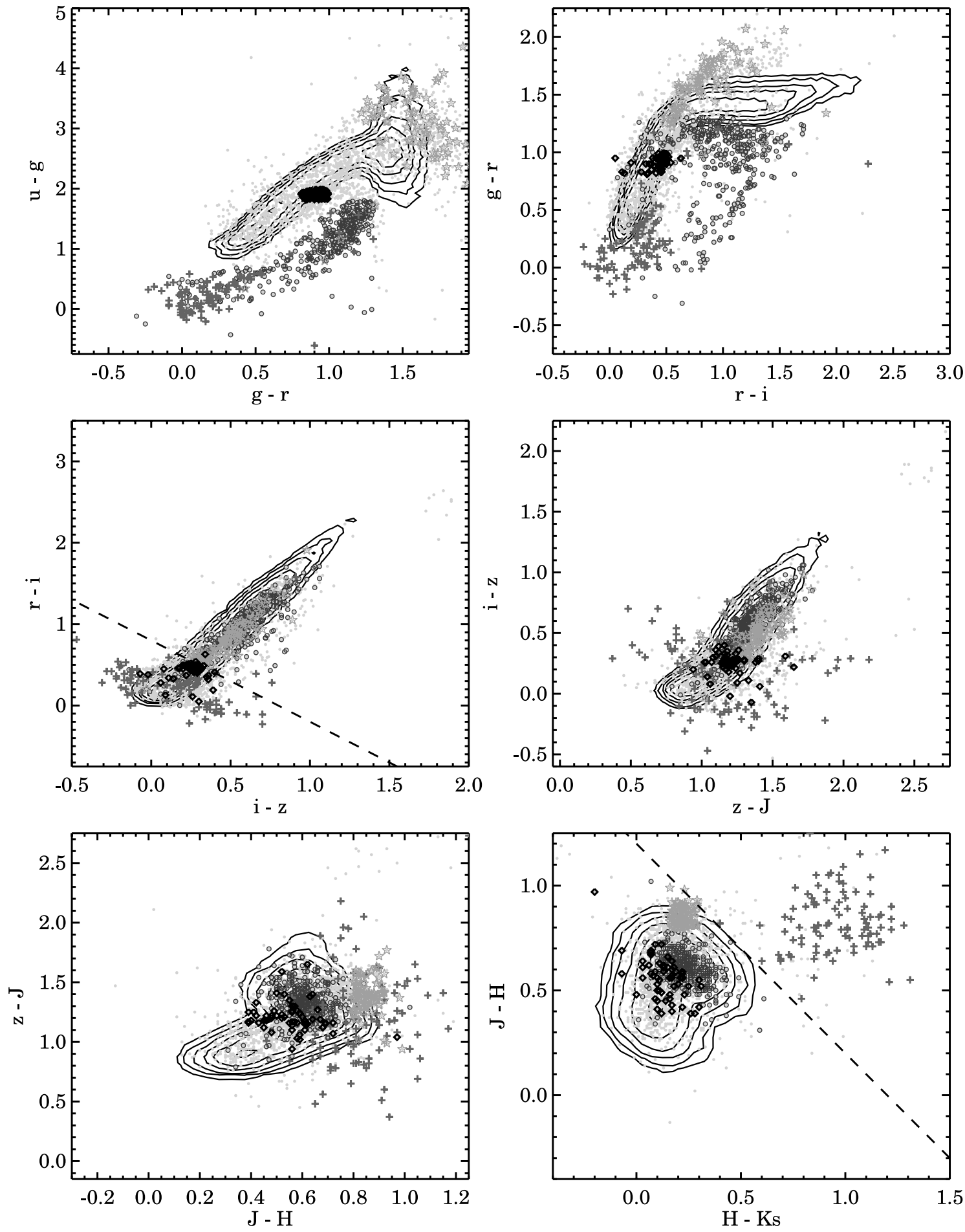

FIG. 13.- Location in color space of 2117 high-quality color outliers in our sample ( gray dots), compared to the location of the SDSS/2MASS stellar locus, shown with black dots and contours. Objects selected as likely QSOs, WDMD pairs, or M giants/carbon stars are shown as crosses, circles, or stars, respectively. Diamonds identify a subset of outliers that blend into the margin of the stellar locus; selected on the basis of their $u-g$ and $g-r$ colors, these objects nevertheless show a consistent offset in $r-i$ as well. Dashed lines indicate the $r-z=0.8$ and $J-K=1.2$ cuts useful for separating QSOs and WDMD pairs. [See the electronic edition of the Journal for a color version of this figure.]

$<0.6^{\prime \prime}$ and CHILD $=0$ to filter out objects whose anomalous colors are merely the result of spurious catalog matches.

As a final test of the utility of the 7DCD, we examine the extent to which each survey contributes to the identification of a source as a color outlier. Figure 12 shows that for the point sources included in our sample (high-latitude point sources with highquality detections in both SDSS and 2MASS), SDSS provides the bulk of the leverage for identifying color outliers. In particular, if each filter contributed an equal amount of signal to the 7DCD, we would expect the typical source to have an SDSS-only color distance equal to $76 \%$ of the total 7DCD. However, color distances computed from SDSS colors alone are $\geq 76 \%$ of the 7DCD for $84.6 \%$ of the sources with 7 DCD $>6$. Similarly, the median SDSS-only color distance is $90 \%$ of the median 7DCD for sources 
with 7DCDs $>6$, suggesting that SDSS colors make up most of the signal captured in the 7DCDs of color outliers.

As we demonstrate below, the addition of $J H K_{s}$ photometry can assist in the classification of certain classes of objects identified as color outliers on the basis of SDSS photometry, but it does not appear to identify new classes of outliers which otherwise appear normal in SDSS photometry. This may change in the near future, as UKIDSS photometry will provide NIR colors with smaller errors and a better match to the dynamic range and spatial resolution of the SDSS, potentially uncovering new classes of faint objects with odd optical - near infrared colors. For now, however, cataloged SDSS-2MASS point sources are most useful for characterizing main-sequence stars along the stellar locus, or for studying "drop-out" objects, such as late L and T dwarfs, which appear in 2MASS but may be detected only in the SDSS $z$ band (Metchev et al. 2007).

\subsection{Properties of Identified Color Outliers}

Having verified the utility of the 7DCD parameter, and motivated by the change in slope seen at $7 \mathrm{DCD}=6$ in Figure 6 , we have assembled a catalog of 2117 color outliers $(0.31 \%$ of the original matched SDSS/2MASS sample) with 7DCDs $>6$, astrometric matching distances $<0.6^{\prime \prime}$, and CHILD $=0$. As Figure 13 shows, the large 7DCD sample makes concentrations of objects with unusual colors, such as QSOs and unresolved white-dwarf/ M dwarf pairs (WDMDs), clearly visible.

Using color constraints we provide preliminary classifications for $25 \%$ of the objects with large 7DCDs. In particular, a pair of $u-g$ versus $g-r$ color cuts $[u-g<0.7$ and $g-r<0.55$ or $0.55 \geq g-r \leq 1.3$ and $u-g<-0.4+2 \times(g-r)]$ identify 463 objects within the region of $u-g$ versus $g-r$ color-color space inhabited by QSOs and WDMDs. While these objects are easily identified on the basis of their unusual $u-g$ versus $g-r$ colors, the blue end of the WDMD locus overlaps with the region typically inhabited by QSOs, making the two classes difficult to distinguish photometrically in $u-g$ versus $g-r$ color-color space. As shown in Figure 13, however, they do possess distinct $J-K_{s}$ and, to a lesser extent, $r-z$ colors; QSOs typically have $J-K_{s}>1.2$ and $r-z<0.8$ and WDMD pairs the opposite. On the basis of their $J-K_{s}$ colors, we are able to identify 93 of these outliers as candidate QSOs and 370 as candidate WDMD pairs.

The last class of outliers we identify are 90 objects with NIR colors typical of $\mathrm{M}$ giants or carbon stars $(J-H>0.8$ and $0.15<$ $\left.H-K_{s}<0.3\right)$. While these sources are most simply identified on the basis of their NIR colors, Figure 13 shows that most are outside the $g-r$ versus $r-i$ stellar locus, and offset relative to the median $r-i$ versus $i-z$ locus as well; distinguishing such sources from other objects along the edge of the stellar locus, however, would be difficult on the basis of their optical SDSS photometry alone.

The majority of the color outliers detected in this catalog, however, appear to be located along the outskirts of the stellar locus. As an example, we highlight a cluster at $u-g \sim 1.9$ and $g-r \sim 0.9$ in Figure 13. These sources, identified solely on the basis of their $u-g$ and $g-r$ colors, form a coherent, distinctive clump in $g-r$ versus $r-i$ color-color space as well; originally identified as sources with $g-r$ colors redward of most stars with the same $u-g$ color, their $g-r$ colors are consistently blueward of sources with similar $r-i$ colors. A preliminary search of the SDSS spectroscopic database fails to identify any of these objects with SDSS spectra, so additional spectroscopic programs will be required to identify the cause of the correlated color offsets dis- played by these sources. Their identification, however, demonstrates the utility of the 7DCD for revealing the presence of objects with small, but consistent, offsets in color-color space.

\section{SUMMARY AND CONCLUSIONS}

Using a sample of more than 600,000 point sources detected in SDSS and 2MASS, we have traced the location of mainsequence stars through ugrizJHK $K_{s}$ color-color space, parameterizing and tabulating the position and width of the stellar locus as a function of $g-i$ color. To provide context for this 1D representation of the stellar locus, we have used synthetic photometry of spectral atlases, as well as analysis of 3000 SDSS stellar spectra by a custom spectral typing pipeline (the Hammer) to produce estimates of stellar ugrizJHK colors and absolute $J$ magnitude $\left(M_{J}\right)$ as a function of spectral type. These measurements will provide guidance for those seeking to interpret the millions of stars detected in SDSS and 2MASS, as well as in future surveys (such as UKIDSS, Pan-STARRS, and SkyMapper) making use of similar filter sets.

We have also developed an algorithm to calculate a point source's minimum separation in color space from the stellar locus. This parameter, which we identify as an object's seven-dimensional color distance, accounts for the intrinsic width of the stellar locus, as well as photometric errors, and provides a robust identification of objects in unique areas of color space. Reliability tests reveal the basic utility of the color distance parameter for identifying color outliers, but also identify spurious SDSS/2MASS matches (typically with SDSS/2MASS astrometric separations $>0.6^{\prime \prime}$ or the SDSS CHILD flag set) as a source of erroneously large color distances. Analysis of a final catalog of 2117 color outliers identified as having color-distances $>6$ identifies 370 white-dwarf/M dwarf pairs, 93 QSOs, and $90 \mathrm{M}$ giant/carbon star candidates, and demonstrates how WDMD pairs and QSOs can be distinguished on the basis of their $J-K_{s}$ and $r-z$ colors. A group of objects with correlated offsets in both the $u-g$ versus $g-r$ and $g-r$ versus $r-i$ color-color spaces is also identified as deserving of subsequent follow-up. Future applications of this algorithm to a matched SDSS-UKIDSS catalog may identify additional classes of objects with unusual colors by probing new areas of color-magnitude space.

Funding for the SDSS and SDSS-II has been provided by the Alfred P. Sloan Foundation, the Participating Institutions, the National Science Foundation, the US Department of Energy, the National Aeronautics and Space Administration, the Japanese Monbukagakusho, the Max Planck Society, and the Higher Education Funding Council for England. The SDSS Web site is at http://www.sdss.org.

The SDSS is managed by the Astrophysical Research Consortium for the Participating Institutions. The Participating Institutions are the American Museum of Natural History, Astrophysical Institute Potsdam, the University of Basel, the University of Cambridge, Case Western Reserve University, the University of Chicago, Drexel University, Fermilab, the Institute for Advanced Study, the Japan Participation Group, Johns Hopkins University, the Joint Institute for Nuclear Astrophysics, the Kavli Institute for Particle Astrophysics and Cosmology, the Korean Scientist Group, the Chinese Academy of Sciences (LAMOST), Los Alamos National Laboratory, the Max Planck Institute for Astronomy, the Max Planck Institute for Astrophysics, New Mexico State University, Ohio State University, the University of Pittsburgh, the 
University of Portsmouth, Princeton University, the US Naval Observatory, and the University of Washington.

The Two Micron All Sky Survey was a joint project of the University of Massachusetts and the Infrared Processing and Analysis Center (California Institute of Technology). The University of Massachusetts was responsible for the overall management of the project, the observing facilities, and the data acquisition. The Infrared Processing and Analysis Center was responsible for data processing, data distribution, and data archiving.

This research has made use of NASA's Astrophysics Data System Bibliographic Services, the SIMBAD database, operated at CDS, Strasbourg, France, and the VizieR database of astronomical catalogs (Ochsenbein et al. 2000). Support for this work was provided by NASA through the Spitzer Space Telescope Fellowship Program, through a contract issued by the Jet Propulsion Laboratory, California Institute of Technology, under a contract with NASA. K. R. C. also gratefully acknowledges the support of NASA grant 80-0273 during the initial stages of this work.

\section{APPENDIX A}

\section{THE HAMMER - AN IDL-BASED SPECTRAL TYPING SUITE}

The Hammer spectral typing algorithm was originally developed for use on late-type SDSS spectra, but has subsequently been modified to allow it to classify spectra in a variety of formats with targets spanning the MK spectral sequence. In this appendix we document the Hammer's index set and describe the algorithm employed to automatically assign spectral types to input target spectra. Also, we provide a brief discussion of the use of the program, which includes an interactive mode which allows the user to assign final spectral types via visual comparison with a grid of spectral templates. We conclude this appendix with a discussion of tests of the accuracy of the Hammer, as well as a few caveats concerning its limitations. This code has been made available for community use, with an archival version available from the Astronomical Journal in the electronic edition of this article. Updated versions of the Hammer can also be downloaded from the Hammer's Web site. ${ }^{18}$

\section{A1. SPECTRAL INDICES}

To estimate the spectral type of an input spectrum, the Hammer measures a set of 26 atomic ( $\mathrm{H}, \mathrm{Ca}$ I, Ca II, $\mathrm{Na}$ I, $\mathrm{Mg}$ I, Fe I, Rb, and $\mathrm{Cs}$ ) and molecular ( $\mathrm{G}$ band, $\mathrm{CaH}, \mathrm{TiO}, \mathrm{VO}$, and $\mathrm{CrH}$ ) features that are prominent in late-type stars, as well as two colors (BlueColor and Color-1) that sample the broadband shape of the SED. These indices sample spectral features strong enough to be visible in moderate-resolution spectra and that have previously been shown to be sensitive to photospheric temperature (Faber et al. 1985; Gorgas et al. 1993; Torres-Dodgen \& Weaver 1993; Worthey et al. 1994; Allen \& Strom 1995; Schmidt-Kaler \& Malyuto 1996; Malyuto et al. 1997; Malyuto \& Schmidt-Kaler 1997, 1999; Gizis \& Reid 1999; Kirkpatrick et al. 1999; Morrison et al. 2003; Ivanov et al. 2004).

These spectral features are measured with ratios of the mean flux density within different spectral bandpasses, such that

$$
\text { Index }=\frac{\text { Mean Flux Density }(\mathrm{N})}{\text { Mean Flux Density(D) }}
$$

\footnotetext{
${ }^{18}$ See http://www.cfa.harvard.edu/ kcovey/thehammer.
}

TABLE 6

Single Numerator Hammer Spectral Indices

\begin{tabular}{|c|c|c|c|c|}
\hline Spectral Feature & $\begin{array}{c}\text { N Start } \\
(\AA)\end{array}$ & $\begin{array}{l}\text { N End } \\
(\AA)\end{array}$ & $\begin{array}{c}\text { D Start } \\
(\AA)\end{array}$ & $\begin{array}{c}\text { D End } \\
(\AA)\end{array}$ \\
\hline $\mathrm{Ca} \mathrm{K}$ & 3923.7 & 3943.7 & 3943.7 & 3953.7 \\
\hline $\mathrm{H} \delta$ & 4086.7 & 4116.7 & 4136.7 & 4176.7 \\
\hline Ca I $\lambda 4227 \ldots \ldots \ldots \ldots . .$. & 4216.7 & 4236.7 & 4236.7 & 4256.7 \\
\hline 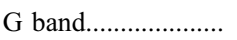 & 4285.0 & 4315.0 & 4260.0 & 4285.0 \\
\hline $\mathrm{H} \gamma$ & 4332.5 & 4347.5 & 4355.0 & 4370.0 \\
\hline $\mathrm{Fe}_{\mathrm{I}} \lambda 4383 \ldots \ldots \ldots \ldots$ & 4378.6 & 4388.6 & 4355.0 & 4370.0 \\
\hline $\mathrm{Fe}_{\text {I }} \lambda 4405 \ldots \ldots \ldots \ldots$ & 4399.8 & 4409.8 & 4414.8 & 4424.8 \\
\hline BlueColor ................... & 6100.0 & 6300.0 & 4500.0 & 4700.0 \\
\hline $\mathrm{H} \beta$ & 4847.0 & 4877.0 & 4817.0 & 4847.0 \\
\hline Mg I $\lambda 5172 \ldots \ldots \ldots \ldots . .$. & 5152.7 & 5192.7 & 5100.0 & 5150.0 \\
\hline $\mathrm{Na} \mathrm{D}$ & 5880.0 & 5905.0 & 5910.0 & 5935.0 \\
\hline Сa I $\lambda 6162 \ldots \ldots \ldots \ldots . .$. & 6150.0 & 6175.0 & 6120.0 & 6145.0 \\
\hline $\mathrm{H} \alpha$ & 6548.0 & 6578.0 & 6583.0 & 6613.0 \\
\hline $\mathrm{CaH} 3 \ldots \ldots \ldots \ldots \ldots \ldots$ & 6960.0 & 6990.0 & 7042.0 & 7046.0 \\
\hline 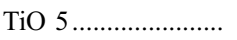 & 7126.0 & 7135.0 & 7042.0 & 7046.0 \\
\hline VO $\lambda 7434 \ldots \ldots \ldots \ldots . .$. & 7430.0 & 7470.0 & 7550.0 & 7570.0 \\
\hline VO $\lambda 7912 \ldots \ldots \ldots \ldots . .$. & 7900.0 & 7980.0 & 8100.0 & 8150.0 \\
\hline 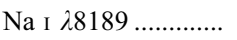 & 8177.0 & 8201.0 & 8151.0 & 8175.0 \\
\hline 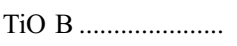 & 8400.0 & 8415.0 & 8455.0 & 8470.0 \\
\hline $\mathrm{TiO} \quad \lambda 8440 \ldots \ldots \ldots \ldots . . .$. & 8440.0 & 8470.0 & 8400.0 & 8420.0 \\
\hline Са II $28498 \ldots \ldots \ldots \ldots . . . .$. & 8483.0 & 8513.0 & 8513.0 & 8543.0 \\
\hline $\mathrm{CrH}-\mathrm{a}$ & 8580.0 & 8600.0 & 8621.0 & 8641.0 \\
\hline Cа II $28662 .$. & 8650.0 & 8675.0 & 8625.0 & 8650.0 \\
\hline Fe I $28689 \ldots \ldots \ldots \ldots$ & 8684.0 & 8694.0 & 8664.0 & 8674.0 \\
\hline Color-1 …...................... & 8900.0 & 9100.0 & 7350.0 & 7550.0 \\
\hline
\end{tabular}

where $\mathrm{N}$ and $\mathrm{D}$ are spectral regions bounded by wavelengths given in Table 6. Table 7 summarizes similar information for four indices where the numerator of the spectral index combines information from multiple bandpasses. For these indices, $\mathrm{N}$ is calculated as

$$
\begin{aligned}
\mathrm{N}= & \mathrm{N}_{1} \text { Weight } \times \text { Mean Flux Density }\left(\mathrm{N}_{1}\right) \\
& +\mathrm{N}_{2} \text { Weight } \times \text { Mean Flux Density }\left(\mathrm{N}_{2}\right),
\end{aligned}
$$

where Table 7 gives the wavelength boundaries and weights of $\mathrm{N}_{1}$ and $\mathrm{N}_{2}$.

To provide a consistent estimate of the uncertainty for both types of spectral indices, we adopt as a characteristic uncertainty the change in the index induced by the uncertainty in the mean flux density of the denominator. This index uncertainty, $\sigma_{\text {Index }}$, is calculated as

$$
\sigma_{\text {Index }}=\sqrt{\left[\operatorname{Index}-\frac{\operatorname{Mean} \operatorname{Flux} \operatorname{Density}(\mathrm{N})}{\operatorname{Mean} \operatorname{Flux} \operatorname{Density}(\mathrm{D})+\sigma_{D}}\right]^{2}},
$$

where $\sigma_{D}$ is the standard deviation of the flux density divided by the number of pixels used to sample that regime, or

$$
\sigma_{D}=\frac{\text { Standard Deviation }(\mathrm{D})}{n_{\mathrm{pix}}}
$$

To map out their variation as a function of spectral type, we have measured these indices in 594 dwarf standards taken from the spectral libraries of Pickles (1998), Hawley et al. (2002), Valdes et al. (2004), Le Borgne et al. (2003), Sanchez-Blazquez et al. (2006), and Bochanski et al. (2007) spanning a range in spectral type from O5 to L8. We measured the median value of 
TABLE 7

Multiple Numerator Hammer Spectral Indices

\begin{tabular}{|c|c|c|c|c|c|c|c|c|}
\hline Spectral Feature & $\begin{array}{c}\text { Num. } 1 \text { Start } \\
(\AA)\end{array}$ & $\begin{array}{l}\text { Num. } 1 \text { End } \\
(\AA)\end{array}$ & Num. 1 Weight & $\begin{array}{c}\text { Num. } 2 \text { Start } \\
(\AA)\end{array}$ & $\begin{array}{l}\text { Num. } 2 \text { End } \\
(\AA)\end{array}$ & Num. 2 Weight & $\begin{array}{c}\text { Denom. Start } \\
(\AA)\end{array}$ & $\begin{array}{l}\text { Denom. End } \\
(\AA)\end{array}$ \\
\hline 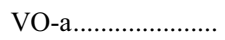 & 7350.0 & 7400.0 & 0.5625 & 7510.0 & 7560.0 & 0.4375 & 7420.0 & 7470.0 \\
\hline VO-b ...................... & 7860.0 & 7880.0 & 0.5 & 8080.0 & 8100.0 & 0.5 & 7960.0 & 8000.0 \\
\hline 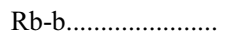 & 7922.6 & 7932.6 & 0.5 & 7962.6 & 7972.6 & 0.5 & 7942.6 & 7952.6 \\
\hline Cs-a …..................... & 8496.1 & 8506.1 & 0.5 & 8536.1 & 8546.1 & 0.5 & 8516.1 & 8526.1 \\
\hline
\end{tabular}

each index as a function of spectral type, linearly interpolating across gaps in the spectral type grid. The nonuniform spectral coverage of these libraries result in some indices being measured reliably only over a restricted range of spectral types; spectra of the reddest stars, for instance, either do not extend to $\mathrm{Ca} \mathrm{K}$, or are too noisy to produce reliable measurements. To compensate for this, when necessary we extended the index values from the earliest and latest templates which produced reliable measurements to the edges of the full spectral grid. Figure 14 displays the resulting median spectral type/spectral index relationships, with the values measured from each individual template also shown for comparison.

\section{A2. AUTOMATED AND INTERACTIVE SPECTRAL TYPE DETERMINATION}

Using these measured spectral type/spectral index relationships, the Hammer generates an automatic estimate of the spectral type of an input target spectrum. To produce this estimate, the Hammer first measures all spectral indices in the above set that are contained within the wavelength coverage of the target spectrum. Spectral indices which are not available in a given target spectrum are flagged as being inaccessible, and are excluded from subsequent analysis.

Each index, however, is most useful within a given spectral type range, and can reduce the accuracy of the spectral typing routine outside that range if it contributes an inordinate amount of weight to the goodness of fit parameter. For this reason, the Hammer excludes indices which are unlikely to be useful in constraining the spectral type of a given input star. To decide which indices to exclude, the Hammer measures the mean wavelength of the target spectrum between 5000 and $7750 \AA$ with and without weighting each wavelength by the flux at that wavelength. The ratio of these means (weighted: nonweighted) is a crude indicator of the shape of a star's SED; late-type stars have more flux at longer wavelengths, and so possess ratios greater than 1, while early-type stars have more flux at short wavelengths, so have ratios less than 1 . For the latest type stars ( $\sim \mathrm{M} 3$ or later; mean wavelength ratios of 1.03 or larger), the Hammer excludes indices tuned for earlytype stars (Ca K, H $\delta, \mathrm{Ca}$ I $\lambda 4227, \mathrm{H} \gamma, \mathrm{Fe}_{\text {I }} \lambda 4383, \mathrm{Fe}$ I $\lambda 4404$, BlueColor, $\mathrm{H} \beta, \mathrm{Na} \mathrm{D}, \mathrm{Ca}$ I $\lambda 6162, \mathrm{H} \alpha, \mathrm{Ca}$ II $\lambda 8498$, and $\mathrm{Fe}$ I 28689). For the earliest type stars ( $\sim \mathrm{F} 0$ and earlier, mean wavelength ratios of 0.97 or less), the Hammer excludes indices useful at cooler temperatures (Ca I $\lambda 4227, \mathrm{Na}$ D, VO $\lambda 7434$, VO-a, VO-b, VO 7912, Rb-b, TiOB, TiO $\lambda 8440$, Cs-a, Ca II $\lambda 8498$, $\mathrm{CrH}-\mathrm{a}, \mathrm{Ca}$ II $28662, \mathrm{Fe}$ г $\lambda 8689$, and color-1). For intermediatetype stars (mean wavelength ratios between 0.97 and 1.03), the Hammer uses all available indices except for $\mathrm{H} \delta$ and $\mathrm{H} \beta$.

The values of the target star's remaining indices are then compared to those of the template grid to determine the best-fit spectral type. Specifically, the differences in the target's indices and the median values associated with a given spectral type are normalized by the errors associated with each index in the target spectrum. After comparing the target indices with the full grid of indices measured from known standards, the Hammer selects the best-fit spectral type by selecting the spectral type associated with the smallest mean squared error-normalized residuals.

Once the Hammer has produced an initial estimate of the spectral type of each input spectrum, it enters an interactive mode whereby the user can perform a direct visual comparison of the target spectrum to a grid of spectral templates. In this mode, the user can smooth the target spectrum for clarity, assign a final MK spectral type, mark a spectrum as hopelessly noisy ("bad"), or assign it a three-character flag for later identification (the "odd" button). This routine also allows the user to save the results of interactive typing before the full list of input stars have been processed (the "break" button), and saves both the automated and interactive spectral type assigned to each spectrum. Although originally developed to process SDSS spectra, the Hammer has since been modified to allow it to process spectra in a variety of formats, and also incorporates routines written by West et al. (2004) to identify M-type subdwarfs and diagnose stellar magnetic activity.

\section{A3. SPECTRAL TYPING ACCURACY AND CAVEATS}

The robustness of the Hammer's spectral typing algorithm has been tested by measuring the errors in the spectral types it assigns to dwarf templates of known spectral type, synthetically degraded with Gaussian white noise to $\mathrm{S} / \mathrm{N} \sim 5$. These tests indicate that the Hammer's automated spectral types are accurate to within \pm 2 subclasses for $\mathrm{K}$ and $\mathrm{M}$ type stars, the regime for which the Hammer has been optimized. At warmer temperatures, the Hammer is somewhat less accurate; typical uncertainties of \pm 4 subtypes are found for $\mathrm{A}-\mathrm{G}$ stars at $\mathrm{S} / \mathrm{N} \sim 5$.

We note, however, two caveats concerning the utility of the Hammer as a spectral typing engine. First, the mapping of index strength as a function of spectral type on which the Hammer is constructed has only been calibrated for solar-metallicity dwarf stars. As a result, the Hammer cannot produce accurate spectral types for stars outside this regime of metallicity and luminosity class; spectral types derived for nonsolar, nondwarf stars will be prone to significant, systematic errors.

Second, the Hammer was originally designed to process stellar spectra obtained by SDSS-I at high Galactic latitude. As the extinction toward these stars is typically very small, and the spectrophotometry produced by the SDSS pipeline is accurate to within a few percent, the Hammer has been designed to make use of the information contained in the slope of the stellar continuum, both in the form of specific indices (BlueColor, Color-1) and in using the SED slope to determine the most useful index set for a given target. As a result, the Hammer will not produce reliable results for spectra where the intrinsic slope of the stellar continuum has not been preserved, either due to instrumental effects or the presence of significant amounts of extinction. For spectra such as these, spectral types should be assigned with methods less sensitive to the shape of the stellar continuum. 

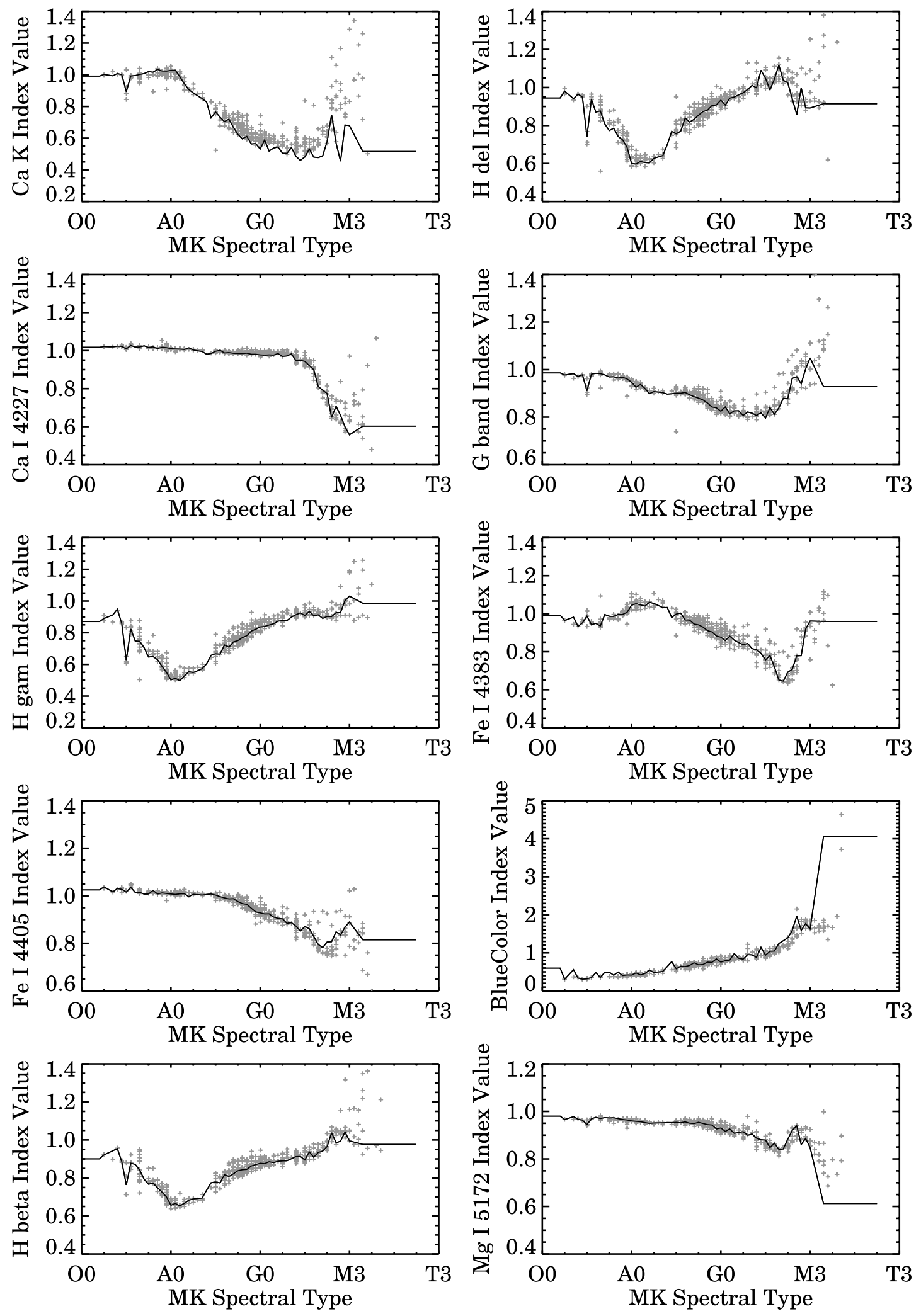

FIG. 14.- Variations of spectral indices as a function of MK spectral type. Gray crosses denote the spectral index as measured for a single spectral type template; the solid line gives the median index vs. spectral type relation used by the Hammer. 

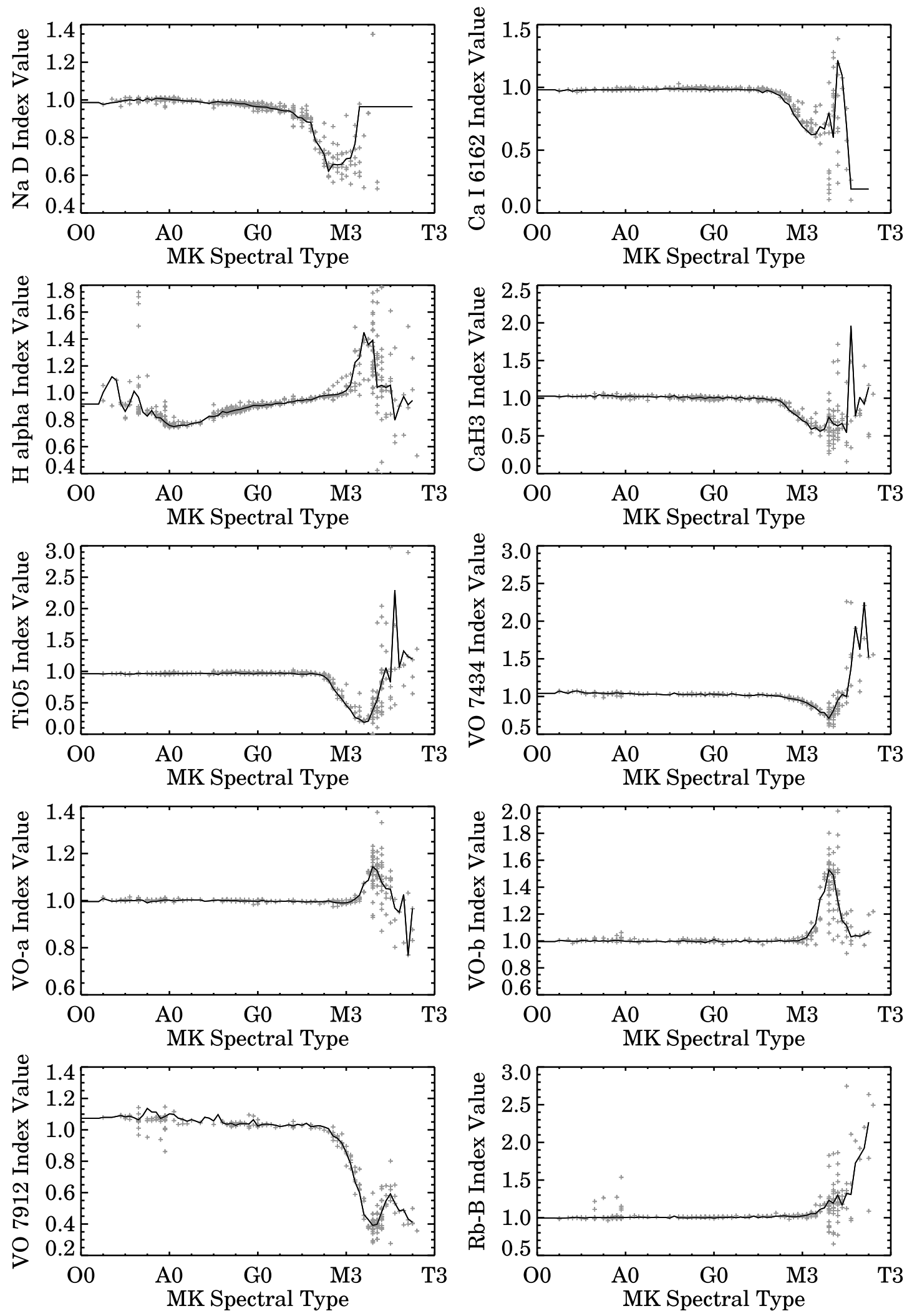

FIG. 14-Continued 

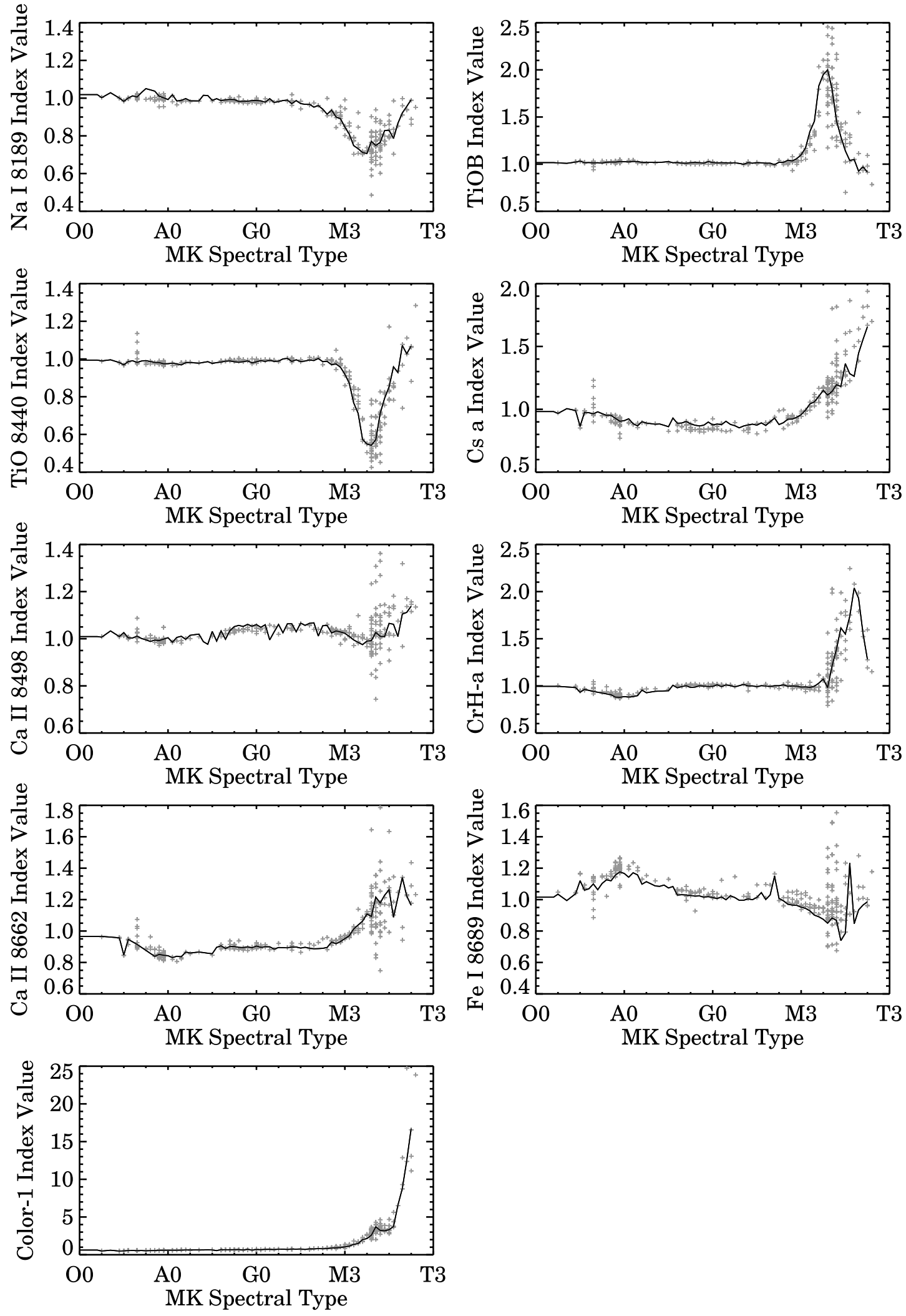

FIG. 14-Continued 
Abazajian, K., et al. 2004, AJ, 128, 502

Adelman-McCarthy, J. K., et al. 2007, ApJS, 172, 634

Agüeros, M. A., et al. 2005, AJ, 130, 1022

Allen, L. E., \& Strom, K. M. 1995, AJ, 109, 1379

Anderson, S. F., et al. 2007, AJ, 133, 313

Bilir, S., Karaali, S., \& Tunçel, S. 2005, Astron. Nachr., 326, 321

Bochanski, J. J., West, A. A., Hawley, S. L., \& Covey, K. R. 2007, AJ, 133, 531

Burgasser, A. J., et al. 1999, ApJ, 522, L65

Carney, B. W., Latham, D. W., Stefanik, R. P., Laird, J. B., \& Morse, J. A. 2003, AJ, 125, 293

Carpenter, J. M. 2001, AJ, 121, 2851

Cohen, M., Wheaton, W. A., \& Megeath, S. T. 2003, AJ, 126, 1090

Cutri, R. M., et al. 2003, 2MASS All Sky Catalog of Point Sources (Greenbelt: NASA)

Davenport, J. R. A., West, A. A., Matthiesen, C. K., Schmieding, M., \& Kobelski, A. 2006, PASP, 118, 1679

Delfosse, X., et al. 2004, in ASP Conf. Ser. 318: Spectroscopically and Spatially Resolving the Components of the Close Binary Stars, ed. R. W. Hilditch, H. Hensberge, \& K. Pavlovski (San Francisco: ASP), 166

Dotter, A. L., et al. 2007, AJ, 134, 376

Duquennoy, A., \& Mayor, M. 1991, A\&A, 248, 485

Eisenstein, D. J., et al. 2006, ApJS, 167, 40

Faber, S. M., Friel, E. D., Burstein, D., \& Gaskell, C.M. 1985, ApJS, 57, 711

Finkbeiner, D. P., Schlegel, D. J., Gunn, J. E., Hogg, D. W., Ivezic, Z., Knapp, G. R., \& Lupton, R. H. 2004a, BAAS, 36, 770

Finkbeiner, D. P., et al. 2004b, AJ, 128, 2577

Finlator, K., et al. 2000, AJ, 120, 2615

Fukugita, M., Ichikawa, T., Gunn, J. E., Doi, M., Shimasaku, K., \& Schneider, D. P. $1996, \mathrm{AJ}, 111,1748$

Girardi, L., Grebel, E. K., Odenkirchen, M., \& Chiosi, C. 2004, A\&A, 422, 205

Gizis, J. E., \& Reid, I. N. 1999, AJ, 117, 508

Gorgas, J., Faber, S. M., Burstein, D., Gonzalez, J. J., Courteau, S., \& Prosser, C. 1993, ApJS, 86, 153

Gunn, J. E., Siegmund, W. A., \& Mannery, E. J. 2006, AJ, 131, 2332

Gunn, J. E., et al. 1998, AJ, 116, 3040

Hawley, S. L., et al. 2002, AJ, 123, 3409

Hogg, D. W., Finkbeiner, D. P., Schlegel, D. J., \& Gunn, J. E. 2001, AJ, 122, 2129

Ivanov, V. D., Rieke, M. J., Engelbracht, C. W., Alonso-Herrero, A., Rieke, G. H., \& Luhman, K. L. 2004, ApJS, 151, 387

Ivezić, Z., Vivas, A. K., Lupton, R. H., \& Zinn, R. 2005, AJ, 129, 1096

Ivezić, Ž., et al. 2002, in IAU Colloq. 184, AGN Surveys, ed. R. F. Green, E. Y.

Khachikian, \& D. B. Sanders (ASP Conf. Ser. 284; San Francisco: ASP), 137 2004, Astron. Nachr., 325, 583

Kaiser, N., et al. 2002, Proc. SPIE, 4836, 154

Keller, S. C., et al. 2007, preprint (arXiv: 0704.1339v1)

\section{RFERENCES}

Kirkpatrick, J. D., et al. 1999, ApJ, 519, 802

Kurucz, R. L. 1979, ApJS, 40, 1

Le Borgne, J.-F., et al. 2003, A\&A, 402, 433

Lupton, R., Gunn, J. E., Ivezić, Z., Knapp, G. R., Kent, S., \& Yasuda, N. 2001, in ASP Conf. Ser. 238, Astronomical Data Analysis Software and Systems X, ed. F. R. Harnden, Jr., et al. (San Francisco: ASP), 269

Malyuto, V., Oestreicher, M. O., \& Schmidt-Kaler, T. 1997, MNRAS, 286, 500

Malyuto, V., \& Schmidt-Kaler, T. 1997, A\&A, 325, 693 1999, Astron. Nachr., 320, 71

Margon, B., et al. 2002, AJ, 124, 1651

Metchev, S., et al. 2007, ApJ, submitted

Morrison, H. L., et al. 2003, AJ, 125, 2502

Newman, P. R., et al. 2004, Proc. SPIE, 5492, 533

Obrić, M., et al. 2006, MNRAS, 370, 1677

Ochsenbein, F., Bauer, P., \& Marcout, J. 2000, A\&AS, 143, 23

Padmanabhan, N., et al. 2007, ApJ, submitted (astro-ph/0703454)

Pickles, A. J. 1998, PASP, 110, 863

Pier, J. R., Munn, J. A., Hindsley, R. B., Hennessy, G. S., Kent, S. M., Lupton, R. H., \& Ivezić, Z. 2003, AJ, 125, 1559

Pourbaix, D., et al. 2005, A\&A, 444, 643

Raymond, S. N., et al. 2003, AJ, 125, 2621

Richards, G. T., et al. 2002, AJ, 123, 2945

Sanchez-Blazquez, P., et al. 2006, MNRAS, 371, 703

Schlegel, D. J., Finkbeiner, D. P., \& Davis, M. 1998, ApJ, 500, 525

Schmidt-Kaler, T., \& Malyuto, V. 1996, A\&AS, 120, 423

Schneider, D. P., et al. 2007, AJ, 134, 102

Scranton, R., et al. 2005, AJ, submitted (astro-ph/0508564)

Silvestri, N. M., et al. 2006, AJ, 131, 1674

Skrutskie, M. F., et al. 1997, in The Impact of Large Scale Near-IR Sky Surveys, ed. F. Garzon et al. (Dordrecht: Kluwer), 25

Smith, J. A., et al. 2002, AJ, 123, 2121

Smolčić, V., et al. 2004, ApJ, 615, L14

Stoughton, C., et al. 2002, AJ, 123, 485

Strauss, M. A., et al. 1999, ApJ, 522, L61

Torres-Dodgen, A. V., \& Weaver, W. B. 1993, PASP, 105, 693

Tucker, D. L., et al. 2006, Astron. Nachr., 327, 821

Valdes, F., Gupta, R., Rose, J. A., Singh, H. P., \& Bell, D. J. 2004, ApJS, 152, 251

Warren, S. J., et al. 2007, MNRAS, 375, 213

West, A. A., Garcia-Appadoo, D. A., Dalcanton, J. J., Disney, M. J., Rockosi,

C. M., Ivezić, Ž., Bentz, C. M., \& Brinkmann, J. 2007, AJ, submitted

West, A. A., Walkowicz, L. M., \& Hawley, S. L. 2005, PASP, 117, 706

West, A. A., et al. 2004, AJ, 128, 426

Williams, C. C., et al. 2002, BAAS, 34, 1292

Worthey, G., Faber, S. M., Gonzalez, J. J., \& Burstein, D. 1994, ApJS, 94, 687

York, D. G., et al. 2000, AJ, 120, 1579 